\title{
The fixed-point property for represented spaces
}

\author{
Mathieu Hoyrup \\ Université de Lorraine, CNRS, Inria, LORIA, F-54000 Nancy, France \\ mathieu.hoyrup@inria.fr
}

January 28, 2021

\begin{abstract}
We investigate which represented spaces enjoy the fixed-point property, which is the property that every continuous multi-valued function has a fixed-point. We study the basic theory of this notion and of its uniform version. We provide a complete characterization of the countable-based $T_{0}$-spaces with the fixed-point property, showing that they are exactly the pointed $\omega$-continuous dcpos. We prove that the spaces whose lattice of open sets enjoys the fixed-point property are exactly the countably-based spaces. While the role played by fixed-point free functions in the diagonal argument is well-known, we show how it can be adapted to fixed-point free multi-valued functions, and apply the technique to identify the base-complexity of the Kleene-Kreisel spaces, which was an open problem. ${ }^{1}$
\end{abstract}

\section{Introduction}

\subsection{The fixed-point property}

A mathematical space $X$ has the fixed-point property if every function $f: X \rightarrow X$ in a certain class has a fixed-point, i.e. some $x \in X$ such that $f(x)=x$. This property plays an important role in several branches of mathematics, witnessed by a number of fixed-point theorems asserting identifying spaces enjoying this property. Among the most celebrated results let us cite Brouwer's fixed-point theorem in topology, Schauder's fixed-point theorem in convex analysis, Knaster-Tarski's and Kleene's fixed-point theorems in order theory, Rogers's fixedpoint theorem (also called the Recursion theorem) in computability theory.

In this article we work in the setting of computable analysis [Wei00], where the main structure is a represented space, and the associated functions are the multi-valued functions (also called multifunctions) that are computable, or more generally continuously realizable (continuous for short). If $X$ is a set and $h: X \rightrightarrows X$ is a multifunction then a point $x \in X$ is a fixed-point of $h$ if $x \in h(x)$.

Therefore, the version of the fixed-point property for computable analysis is the following:

Definition 1.1. A represented space $\mathrm{X}$ has the fixed-point property if every continuous multifunction $h: \mathbf{X} \rightrightarrows \mathbf{X}$ has a fixed-point.

This paper is an investigation of the following question:

Question 1.1. Which represented spaces $\mathbf{X}$ enjoy the fixed-point property?

\footnotetext{
${ }^{1}$ This is the second version of the article with two major changes: we build a space having the fixed-point property but not the uniform one, and we remove the proof of the characterization of Scott topologies on continuous dcpos, which turned out to be already known.
} 
It turns out that most represented spaces satisfying the fixed-point property actually enjoy a stronger, uniform version of it, where a fixed-point can be continuously found from the multifunction. We therefore introduce a uniform version of the fixed-point property, which will prove useful.

We give a first characterization of the uniform fixed-point property, which implies in particular that its computable version is equivalent to the notion of multi-retraceable represented space recently introduced by Brattka and Gherardi in [BG21].

Theorem (Theorem 4.1). Let $\mathbf{X}$ be a represented space. The following conditions are equivalent:

- $\mathbf{X}$ has the (computable) uniform fixed-point property,

- Every (computable) continuous function $f: \subseteq \mathcal{N} \rightarrow \mathbf{X}$ has a total (computable) continuous extension.

We relate the uniform fixed-point property to the notion of effective discontinuity introduced by Brattka in [Bra20] where it is proved, assuming the Axiom of Determinacy, that discontinuity is equivalent to effective discontinuity. It translates into the following result, explaining why in practice, spaces enjoying the fixed-point property actually enjoy its uniform version.

Theorem (Theorem 4.2). Let $\mathbf{X}$ be a represented space. Assuming the Axiom of Determinacy, the following conditions are equivalent:

- $\mathbf{X}$ has the fixed-point property,

- $\mathbf{X}$ has the uniform fixed-point property.

We next give a simple criterion which can be particularly helpful to disprove the fixedpoint property, without having to explicitly build a fixed-point free continuous multifunction (here, $[\Gamma](\mathbf{X})$ is the class of subsets of $\mathbf{X}$ whose preimage under the representation has complexity $\Gamma$, see Definition 2.1).

Theorem (Theorem 4.4). Let $\Gamma$ be a class of the Borel, Hausdorff-Kuratowski or hyperprojective hierarchy on $\mathcal{N}$ which is not self-dual, and assume that equality on $\mathbf{X}$ has complexity $[\Gamma](\mathbf{X} \times \mathbf{X})$. For $A \subseteq \mathbf{X}$ :

- If $A$ has the fixed-point property then $A$ is not $\check{\Gamma}$-hard,

- If $A$ has the uniform fixed-point property then $A \in[\Gamma](\mathbf{X})$.

We then turn our attention to restricted classes of represented spaces, for which we can give an elegant characterization of the fixed-point property and at the same time show its equivalence with the uniform fixed-point property, without assuming the Axiom of Determinacy.

We focus on the class of countably-based $T_{0}$-spaces, on which a subsequent part of computable analysis was first developed (they are central in the book of Weihrauch [Wei00]). We show that among these spaces, the ones with the fixed-point property are exactly the pointed $\omega$ continuous dcpos, a very important class of partial orders studied in domain theory (the statement involves the notion of c-space, which will be explained in due time).

Theorem (Theorem 5.1). Let $\mathbf{X}$ be a countably-based $T_{0}$-space. The following statements are equivalent:

1. $\mathbf{X}$ has the fixed-point property,

2. $\mathbf{X}$ has the uniform fixed-point property, 
3. $\mathbf{X}$ is a multivalued retract of $\mathcal{P}(\omega)$,

4. $\mathbf{X}$ is aquasiPolish c-space with a least element,

5. $\mathbf{X}$ is homeomorphic to a pointed $\omega$-continuous dcpo endowed with the Scott topology.

We also focus on spaces of open sets, on which one can take advantage of the lattice structure to give a very neat characterization of the fixed-point property and again prove its equivalence with its uniform version, without assuming the Axiom of Determinacy.

Theorem (Theorem 6.1). Let $\mathbf{X}$ be admissibly represented. The following conditions are equivalent:

- $\mathbf{X}$ is countably-based,

- $\mathcal{O}(\mathbf{X})$ has the uniform fixed-point property,

- $\mathcal{O}(\mathbf{X})$ has the fixed-point property.

\subsection{Diagonal argument}

In a second part of the article, we give applications of the failure of the fixed-point property. It is well-known that the diagonal argument is related to the fixed-point property, let us give two famous examples:

- Cantor's diagonal argument uses the boolean negation $\neg:\{0,1\} \rightarrow\{0,1\}$ which has no fixed-point to prove that there is no surjection from $X$ to $\{0,1\}^{X}$,

- By contraposition, and letting $\mathcal{P}$ be the class of partial computable functions, Rogers fixedpoint theorem uses the existence of a computable surjection from $\mathbb{N}$ to the computable elements of $\mathcal{P}^{\mathbb{N}}$ to prove that every computable multifunction $f: \mathcal{P} \rightrightarrows \mathcal{P}$ has a fixed-point.

This very general argument has been abstracted by Lawvere in [Law69] using the language of categories.

However, applying the diagonal argument using fixed-point free multifunctions is not always straightforward, because it produces a multifunction rather than a function. We show how to overcome this problem and give a few applications of the technique, in particular we identify the base-complexity of Kleene-Kreisel spaces, which we explain now.

For each $k \in \mathbb{N}$ (actually, for each countable ordinal), one can define the Kleene-Kreisel space $\mathbb{N}\langle k\rangle$ of continuous functionals of order $k$ as follows: $\mathbb{N}\langle 0\rangle=\mathbb{N}$ and $\mathbb{N}\langle k+1\rangle=\mathbb{N}^{\mathbb{N}\langle k\rangle}$, so that $\mathbb{N}\langle 1\rangle=\mathcal{N}$ and $\mathbb{N}\langle 2\rangle=\left(\mathbb{N}^{\mathbb{N}}\right)^{\mathbb{N}}$ for instance.

It is known that the topology on the Kleene-Kreisel spaces is difficult to understand. It is strictly stronger than the compact-open topology [Hyl79]. It was conjectured for a long time that it is zero-dimensional, but Schröder eventually proved in [Sch09b] that it is neither zero-dimensional nor regular. The definition of the topology is implicit, and no explicit characterization is currently known. A way to tackle this problem is to understand the intrinsic difficulty of describing the open sets. This is what the base-complexity of a topological space, introduced in [dBSS16], is about: given a topological space, it measures the minimal descriptive complexity of a subset of $\mathcal{N}$ from which a basis of the topology can be continuously obtained. The complexity is naturally measured in terms of the (hyper)projective hierarchy $\left(\underset{\sim}{\boldsymbol{\Sigma}_{\alpha}}\right)_{\alpha}$ over the countable ordinals $\alpha$.

The base-complexity of $\mathbb{N}\langle\alpha\rangle$ was essentially unknown, except for the natural upper bound: $\mathbb{N}\langle\alpha\rangle$ is $\Sigma_{\alpha+1}^{1}$-based, but no lower bound was known. A related problem is about the structure of 
the base-complexity hierarchy. It is proved in [dBSS16] that the $\underset{\sim}{\boldsymbol{\Sigma}} \alpha^{1}$-based spaces are a proper subclass of the $\Sigma_{\alpha+3}^{1}$-based spaces (Corollary 6.12 in [dBSS16]), but whether the gap can be reduced is left open (Problem 6.2 in [dBSS16]).

Applying our version of the diagonal argument and the existence of a fixed-point free continuous multifunction on $\mathcal{O}(\mathbb{N}\langle\alpha\rangle)$, we prove that for $\alpha \geq 2$ the upper bound on the base-complexity of $\mathbb{N}\langle\alpha\rangle$ is optimal, giving an instance of a space that is $\boldsymbol{\Sigma}_{\alpha+1}^{1}$-based but not $\boldsymbol{\Sigma}_{\alpha}^{1}$-based, which shows that the base-complexity hierarchy does not collapse.

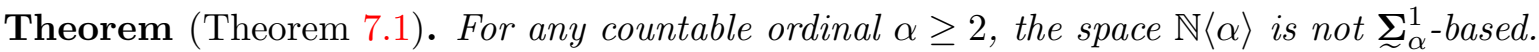

Observe that it contrasts with the case $\alpha=1$, for which $\mathbb{N}\langle 1\rangle=\mathcal{N}$ is countably-based, i.e. $\mathbb{N}\langle 0\rangle$-based.

We also develop the effective version of the main concepts and prove the effective counterpart of the latter result.

Theorem (Theorem 8.1). For $k \in \mathbb{N}$ with $k \geq 2$, there is no $\Sigma_{k}^{1}$-indexing of the effective open subsets of $\mathbb{N}\langle k\rangle$.

We also apply this machinery to identify the indexing complexity of the Markov semidecidable properties of computable functions: their canonical indexing has complexity $\Pi_{3}^{0}$, and this is optimal. This result is not surprising, but is surprisingly difficult to obtain.

Theorem (Theorem 8.2). There is no $\Sigma_{3}^{0}$-indexing of the Markov semidecidable properties of total computable functions.

\subsection{Structure of the article}

The article is organized as follows. In Section 2 we present the needed background about represented spaces and topology. In Section 3 we investigate simple properties of spaces with the fixed-point property. In Section 4 we introduce and study the uniform fixed-point property. In Section 5 we prove the characterization of the countably-based $T_{0}$-spaces with the (uniform) fixed-point property. In Section 6 we prove characterizations of the (uniform) fixed-point property in several classes of spaces: open sets, non-empty closed sets, and functions from Baire space. In Section 7 we show how to apply the diagonal argument using fixed-point free multifunctions in order to determine the base-complexity of Kleene-Kreisel spaces. In Section 8 we investigate the effective version of the previous results, with an application to indexing complexity.

\section{Background}

We give basic definitions and results as well as references on a few different topics: represented spaces, topology, domain theory, Kleene-Kreisel functionals.

\section{$2.1 \quad$ Represented spaces}

We present the necessary definitions about represented spaces, more details can be found in [Wei00, Pau16].

The Baire space is $\mathcal{N}=\mathbb{N}^{\mathbb{N}}$, whose elements are either viewed as functions or infinite sequences. To each finite sequence of natural numbers $\sigma \in \mathbb{N}^{*}$, we associate the cylinder $[\sigma]$ which is the set of elements of $\mathcal{N}$ extending $\sigma$. The Baire space is then endowed with the topology generated by the cylinders. Every subset of $\mathcal{N}$ is endowed with the subspace topology. 
A represented space is a pair $\mathbf{X}=\left(X, \delta_{X}\right)$ where $X$ is a set and $\delta_{X}: \subseteq \mathcal{N} \rightarrow X$ is a partial surjective function called a representation. If $\delta_{X}(p)=x$, then $p$ is a name of $x$. If $\mathbf{X}, \mathbf{Y}$ are represented spaces then a function $F: \subseteq \mathcal{N} \rightarrow \mathcal{N}$ is a realizer of a multifunction $f: \mathbf{X} \rightrightarrows \mathbf{Y}$ if $\delta_{Y}(F(p)) \in f\left(\delta_{X}(p)\right)$ for all $p \in \operatorname{dom}\left(f \circ \delta_{X}\right)$. We say that $f$ is continuous if it has a continuous realizer and that $f$ is computable if it has a computable realizer. We write $\mathbf{X} \cong \mathbf{Y}$ if there exists a bijection between $\mathbf{X}$ and $\mathbf{Y}$ which is computable in both directions.

The next observation is elementary but plays an important role in the diagonal argument.

Proposition 2.1 (Selection on Baire space). Let $\mathbf{X}$ be a represented space. Every continuous multifunction $f: \subseteq \mathcal{N} \rightrightarrows \mathbf{X}$ has a continuous selection, i.e. a continuous function $g: \subseteq \mathcal{N} \rightarrow \mathbf{X}$ satisfying $g(p) \in f(p)$ for all $p \in \operatorname{dom}(f)$.

Proof. The representation of $\mathcal{N}$ is the identity, so every element has a unique name. Therefore, a continuous realizer $F$ of $f$ actually realizes a single-valued function $g=\delta_{X} \circ F$.

There exists a partial computable function $U: \subseteq \mathcal{N} \times \mathcal{N} \rightarrow \mathcal{N}$ that is universal: for every partial continuous function $H: \subseteq \mathcal{N} \rightarrow \mathcal{N}$, there exists $p \in \mathcal{N}$ such that $H=U(p, \cdot)$. We call $p$ a name of $H$.

Admissibility A representation $\delta$ of a topological space $(X, \tau)$ is admissible if $\tau$ is the final topology of $\delta$ and every partial continuous function $f: \subseteq \mathcal{N} \rightarrow X$ has a continuous realizer, which is a continuous function $F: \subseteq \mathcal{N} \rightarrow \mathcal{N}$ satisfying $f=\delta \circ F$.

If $\mathbf{X}, \mathbf{Y}$ are admissibly represented spaces, then a function $f: \mathbf{X} \rightarrow \mathbf{Y}$ is continuous if and only if it has a continuous realizer. In particular, $f$ is continuous if and only if it is computable relative to some oracle.

Note that for multifunctions, no topological characterization of continuity is known, although partial answers have been given in [BH94, PZ13] for instance.

The topological spaces having an admissible representation are exactly the $T_{0}$-spaces that are quotients of countably-based spaces, and are also called $\mathrm{QCB}_{0}$-spaces [Sch02]. These spaces form a cartesian closed category, with very natural representations for products and function spaces $\mathcal{C}(\mathbf{X}, \mathbf{Y})$. When identifying the space of open sets $\mathcal{O}(X)$ with the function space $\mathbb{S}^{X}$ where $\mathbb{S}$ is the Sierpinski space, the induced representation of $\mathcal{O}(X)$ is admissible for the Scott topology (see for instance Proposition 2.2 in [Sch15]).

Countably-based $T_{0}$-spaces have a particular representation, called the standard representation, which is admissible. Once a countable basis indexed by $\mathbb{N}$ has been chosen, say $\left(B_{i}\right)_{i \in \mathbb{N}}$, a name of $x$ is any $p \in \mathcal{N}$ such that $\{i \in \mathbb{N}: \exists n, p(n)=i+1\}=\left\{i \in \mathbb{N}: x \in B_{i}\right\}$, so that $x$ is described by enumerating its basic neighbourhoods in any order.

Descriptive complexity We will only need a few elements of developing descriptive set theory on represented spaces, more on this topic can be found in [PdB15]. Descriptive complexity classes on $\mathcal{N}$ immediately induce classes on any represented space. They are thoroughly studied in [CH20, Hoy20], where they are called symbolic complexity classes.

Definition 2.1 (Symbolic complexity). Let $\Gamma(\mathcal{N})$ be a class of subsets of $\mathcal{N}$ and let $\mathbf{X}$ be a represented space. A set $A \subseteq \mathbf{X}$ belongs to $[\Gamma](\mathbf{X})$ if there exists $S \in \Gamma(\mathcal{N})$ such that

$$
\delta_{X}^{-1}(A)=S \cap \operatorname{dom}\left(\delta_{X}\right) .
$$

A set $A \subseteq \mathbf{X}$ is $\Gamma$-hard if for every $C \in \Gamma(\mathcal{N})$ there exists a continuous function $f: \mathcal{N} \rightarrow \mathbf{X}$ such that $C=f^{1}(A)$. 
It is proved in [dB13] that if $\mathbf{X}$ is a countably-based $T_{0}$-space with the standard representation, then $[\Gamma](\mathbf{X})=\Gamma(\mathbf{X})$ for all the complexity classes from the Borel and HausdorffKuratowski hierarchies. For more general spaces it usually fails as shown in [CH20, Hoy20]. However, hardness is related to symbolic complexity rather than topological complexity: under suitable assumptions about $\mathbf{X}$ and $A \subseteq \mathbf{X}, A \notin[\Gamma](\mathbf{X})$ if and only if $A$ is $\check{\Gamma}$-hard, which is essentially Wadge's Lemma ([Kec95]).

\subsection{Topology}

If $A$ is a subset of a topological space, then $\operatorname{cl}(A)$ denotes its closure and $\operatorname{int}(A)$ denotes its interior.

In a topological space $(X, \tau)$, the specialization pre-order is the relation $\leq$ defined by $x \leq$ $y$ iff every neighborhood of $x$ is a neighborhood of $y$. In other words, $\downarrow y:=\{x \in X: x \leq y\}=$ $\operatorname{cl}(\{y\})$. In a $T_{0}$-space, the specialization pre-order is a partial order. In a $T_{1}$-space, it coincides with equality.

The space $\mathcal{P}(\omega)$ of subsets of $\mathbb{N}$ is endowed with the Scott topology: the sets $\{U \in \mathcal{P}(\omega)$ : $F \subseteq U\}$, where $F$ ranges over the finite sets, is a countable basis of the topology. It is a universal countably-based $T_{0}$-space: a topological space is countably-based and $T_{0}$ if and only

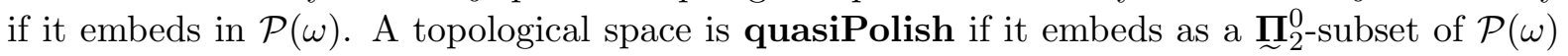
[dB13].

\subsection{Sober spaces and dcpos}

We refer to [GL13] for the interaction between partial orders and topology.

In a topological space, a closed set $C$ is irreducible if it whenever two open sets intersect $C$, their intersection also intersects $C$. A topological space is sober if every irreducible closed set is the closure of a point. Note that the closure of a point $x$ is $\downarrow x=\{y: y \leq x\}$, where $\leq$ is the specialization order.

We will need the following characterization of countably-based sober spaces, analogous to Theorem 8.2.29 in [GL13].

Proposition 2.2. A subspace $X \subseteq \mathcal{P}(\omega)$ is sober if and only if whenever a sequence $\left(x_{n}\right)_{n \in \mathbb{N}} \subseteq$ $X$ converges to $x:=\bigcup_{n} x_{n}, x$ belongs to $X$.

Proof. Assume that $X$ is sober and let $x_{n} \in X$ converge to $x=\bigcup_{n} x_{n}$. The set $C:=X \cap \downarrow x$ is a closed subset of $X$. It is irreducible. If two open sets intersect $C$ then they both contain $x_{n}$ for sufficiently large $n$, and so does their intersection. As $C$ is irreducible, $C$ is the closure of some $y \in X$. One has $y \in C$ so $y \leq x$. As $x_{n} \leq y$ for each $n$, one has $x \leq y$. As a result, $x=y$ so $x \in X$.

Conversely, assume that $X$ satisfies the property and let $C \subseteq X$ be an irreducible closed set. Let $x=\sup C$ in $\mathcal{P}(\omega)$. For each finite set $F \subseteq x$, there exists $y \in C$ containing $F$. Indeed, for each $n \in F$, the open set $\uparrow\{n\}$ intersects $C$, so their finite intersection intersects $C$ by irreducibility. Let $\left(F_{n}\right)_{n \in \mathbb{N}}$ be a growing sequence of finite sets whose union is $x$. For each $n$, there exists $x_{n} \in C$ containing $F_{n}$. Therefore, $x_{n}$ converge to $x=\bigcup_{n} x_{n}$, so $x \in X$. As a result, $C$ is the closure of $x$.

A partial order is a directed-complete partial order (dcpo) if every directed subset has a least upper bound. It is pointed if it has a least element. A subset $U$ of a dcpo is Scott open if it intersects every directed set whose least upper bound belongs to $U$. A function $f$ between dcpos is Scott-continuous if it is monotone and $f(\sup D)=\sup f(D)$ for every directed set. 
In a dcpo, $x$ is way below $y$, written $x \ll y$, if for every directed set $D$, if $y \leq \sup D$ then $x \leq d$ for some $d \in D$. A dcpo is continuous if for each $y$, the set $\downarrow y:=\{x: x \ll y\}$ is directed and $y=\sup \downarrow y$. A continuous dcpo is $\omega$-continuous if its Scott topology is countablybased (it is not the usual definition but it is a characterization, see Lemma 7.7.13 in [GL13]).

c-spaces. The way below relation and the notion of continuity of a dcpo have topological analogs, introduced in [Ern91].

Definition 2.2. Let $(X, \tau)$ be a topological space and $x, y \in X$. Say that $x$ is topologically way below $y$, written $x \ll_{t} y$, if $y$ belongs to the interior of $\uparrow x$.

A topological space $(X, \tau)$ is a c-space if every $y \in X$ belongs to the closure of $\downarrow_{t} y:=\{x \in$ $\left.X: x \ll_{t} y\right\}$.

Note that if $(X, \tau)$ is a c-space, then the closure of $\downarrow_{t} y$ is exactly the closure of $\{y\}$, which is $\downarrow y$. In a c-space, the sets $\uparrow_{t} x$ form a basis of the topology. Every c-space is locally compact, because the sets $\uparrow x$ are compact and induce a local basis of compact neighborhoods at each point.

We will use the next result, which gives a characterization of the Scott topologies on continuous dcpos (Proposition 8.3.36 in [GL13]).

Theorem 2.1. The sober c-spaces are exactly the continuous dcpos endowed with the Scott topology.

\subsection{Kleene-Kreisel functionals}

One defines the admissibly represented space $\mathbb{N}\langle\alpha\rangle$ for all countable ordinal $\alpha$, by transfinite induction. Let $\mathbb{N}\langle 0\rangle=\mathbb{N}, \mathbb{N}\langle\alpha+1\rangle=\mathcal{C}(\mathbb{N}\langle\alpha\rangle, \mathbb{N})$ and $\mathbb{N}\langle\lambda\rangle=\prod_{i \in \mathbb{N}} \mathbb{N}\langle\beta(\lambda, i)\rangle$ for a limit ordinal $\lambda$ and a fixed numbering $\beta(\lambda, i)$ of the ordinals smaller than $\lambda$.

We will use the following results about the Kleene-Kreisel spaces. The first two ones can be found in [Nor80] and were proved by Kleene and Kreisel.

Lemma 2.1. For each $k \in \mathbb{N}, \mathbb{N}\langle k\rangle$ contains a dense computable sequence.

Theorem 2.2 (Theorem 5.22 in [Nor80]). Let $k \in \mathbb{N}$ and $A \subseteq \mathcal{N}$. The following conditions are equivalent:

- $A \in \Sigma_{k}^{1}(\mathcal{N})$,

- There exists a computable predicate $R \subseteq \mathcal{N} \times \mathbb{N}\langle\alpha\rangle \times \mathbb{N}$ such that

$$
x \in A \Longleftrightarrow \exists y \in \mathbb{N}\langle\alpha\rangle \forall n, R(x, y, n) .
$$

In particular, every $\Sigma_{k}^{1}$-set is a computable image of a $\Pi_{1}^{0}$-subset of $\mathbb{N}\langle k\rangle$, but Theorem 2.2 says more and we will need it later.

This result has a non-effective version at all levels of the hyperprojective hierarchy.

Theorem 2.3 (Theorem 6.1 in [SS15b]). Let $\alpha \neq 0$ be a countable ordinal and $A \subseteq \mathcal{N}$ be

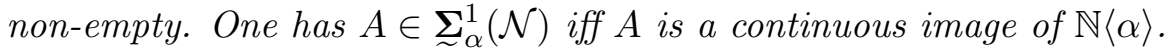

In [SS15a] an admissible representation $\delta_{\alpha}$ of $\mathbb{N}\langle\alpha\rangle$ is built. Let $D_{\alpha}=\operatorname{dom}\left(\delta_{\alpha}\right)$. One has $D_{\alpha+1} \in \prod_{\sim}^{1}(\mathcal{N})$ and $D_{k+1} \in \Pi_{k}^{1}(\mathcal{N})$ for $k \in \mathbb{N}$. 


\section{The fixed-point property}

We start the study of the fixed-point property, introduced at the beginning of Section 1.

It is useful to have the following intuition in mind: a computable fixed-point free multifunction corresponds to an algorithm taking some $x$ as input (represented by a name) and producing some $x^{\prime} \neq x$ as output (which may not be the same for all names of $x$ ).

A simple way to prove that a space has the fixed-point property is to show that it is a retract of another space with the fixed-point property. Multivalued retracts are the right notion in this setting. They also play an important role in [BG21].

Definition 3.1. We say that $\mathbf{Y}$ is a (computable) multivalued retract of $\mathbf{X}$ if there exists a (computable) continuous multifunction $r: \mathbf{X} \rightrightarrows \mathbf{Y}$ and a (computable) continuous function $s$ : $\mathbf{Y} \rightarrow \mathbf{X}$ such that $r \circ s=\mathrm{id}_{\mathbf{Y}}$.

Note in particular that for all $x$ in the image of $s, r(x)$ is a singleton. In particular, $s$ is an embedding of $\mathbf{Y}$ into $\mathbf{X}$, the restriction of $r$ to $s(\mathbf{Y})$ being its inverse.

Remark 3.1. One could also consider a weaker notion of retract, where $s$ is also multivalued. Some of the results still hold for this weaker notion, however $s$ is no more an embedding (for instance, the space $\mathcal{O}(\mathcal{N})$ is not countably-based but it a retract of the countably-based space $\mathcal{P}(\omega)$ for this weaker notion). We will meet this weaker notion in the proof of Theorem 6.1.

Proposition 3.1. Every closed subset of $\mathbf{X}$ is a multivalued retract of $\mathbf{X}$.

Proof. Let $P$ be a closed subset of $\mathbf{X}$ and let $s: P \rightarrow \mathbf{X}$ be the canonical injection. We define $r: \mathbf{X} \rightrightarrows P$ by building a continuous realizer of $r$. Given a name $p$ of $x \in \mathbf{X}$, we start outputting $p$ making sure at each step that the current prefix of the output can be extended to a name of an element of $P$. In parallel, test whether $x \notin P$, which is possible as $P$ is closed. If we eventually see that $x \notin P$, then we extend the current output with a name of an element of $P$. We have $r \circ s=$ id because if $x \in P$ then we simply output $p$, which is a name of $x$.

Proposition 3.2 (Stability under retractions). If $\mathbf{X}$ has the fixed-point property, then every multivalued retract of $\mathbf{X}$ has the fixed-point property. In particular, every closed subset of $\mathbf{X}$ has the fixed-point property.

Proof. If $\mathbf{Y}$ is a a multivalued retract of $\mathbf{X}$, coming with $r, s$, and if $h: \mathbf{Y} \rightrightarrows \mathbf{Y}$ is continuous, then $s \circ h \circ r: \mathbf{X} \rightrightarrows \mathbf{X}$ is continuous so it has a fixed-point $x \in s \circ h \circ r(x)$. It is easily seen that $y=r(x)(r(x)$ is indeed a singleton as $x$ is in the image of $s)$ is a fixed-point of $h$ : one has $x \in s \circ h(y)$ so $y=r(x) \in r \circ s \circ h(y)=h(y)$.

The fixed-point property easily implies the next simple property.

Proposition 3.3 (Least element). If a represented space $\mathbf{X}$ has the fixed-point property, then $\mathbf{X}$ has a least element in the specialization preorder of the final topology of $\delta_{X}$.

A least element is an $x \in \mathbf{X}$ such that $x \leq_{\tau} y$ for all $y \in \mathbf{X}$, and is unique when the space is $T_{0}$. This result immediately excludes every $T_{1}$-space which is not a singleton.

Proof. We assume that $\mathbf{X}$ does not have a least element and build a continuous fixed-point free multifunction $h: \mathbf{X} \rightrightarrows \mathbf{X}$.

Let $E=\left\{\sigma \in \mathbb{N}^{*}: \mathbf{X} \backslash \delta_{X}([\sigma]) \neq \emptyset\right\}$. We first show that every $p \in \operatorname{dom}\left(\delta_{X}\right)$ has a prefix in $E$. Indeed, if $p \in \operatorname{dom}\left(\delta_{X}\right)$ then $x:=\delta_{X}(p)$ is not a least element, i.e. there exists $y \in \mathbf{X}$ 
such that $x \not \leq y$. It means that there exists an open set $U$ containing $x$ but not $y$. Now, $p$ has some prefix $\sigma$ such that $\delta_{X}([\sigma]) \subseteq U$, which implies that $y \notin \delta_{X}([\sigma])$, so $\sigma \in E$.

Using $E$ it is easy to build a continuous fixed-point free multifunction $h: \mathbf{X} \rightrightarrows \mathbf{X}$. Given $p \in$ $\operatorname{dom}\left(\delta_{X}\right)$, find a prefix $\sigma$ of $p$ in $E$, and output (a name of) some $y \in \mathbf{X} \backslash \delta_{X}([\sigma])$, which is necessarily distinct from $\delta_{X}(p)$.

In particular, it implies that if $\mathbf{X}$ has the fixed-point property then $\mathbf{X}$ is compact (again, for the final topology of the representation). A similar result was proved in [BG21] under the stronger assumption that $\mathbf{X}$ is multi-retraceable (we give more information about this notion below).

To a multifunction $f: \mathbf{X} \rightrightarrows \mathbf{Y}$ we associate its adversary function $\mathbb{A}_{f}: \mathbf{Y} \rightrightarrows \mathbf{X}$ defined by $\mathbb{A}_{f}(y)=\{x \in \mathbf{X}: y \notin f(x)\}$. Observe that $\mathbb{A}_{\mathbb{A}_{f}}=f$ and that $\mathbb{A}_{f} \circ f$ and $f \circ \mathbb{A}_{f}$ are fixed-point free.

Proposition 3.4. For a represented space $\mathbf{X}$, the following conditions are equivalent:

- $\mathbf{X}$ has the fixed-point property,

- For every $\mathbf{Y}$ and $f: \mathbf{X} \rightrightarrows \mathbf{Y}, f$ and $\mathbb{A}_{f}$ are not both continuous,

- For every $\mathbf{Y}$ and $f: \mathbf{Y} \rightrightarrows \mathbf{X}, f$ and $\mathbb{A}_{f}$ are not both continuous.

Proof. If $\mathbf{X}$ has the fixed-point property then $\mathbb{A}_{f} \circ f\left(\right.$ or $f \circ \mathbb{A}_{f}$ ), which has no fixed-point, is not continuous so $f$ or $\mathbb{A}_{f}$ is not continuous. Conversely, assuming the second or third condition, the function $f=\mathrm{id}: \mathbf{X} \rightarrow \mathbf{X}$ is continuous so $\mathbb{A}_{f}$ is not, which means that there is no continuous fixed-point free multifunction on $\mathbf{X}$, i.e. that $\mathbf{X}$ has the fixed-point property.

This result has an interesting consequence. Let $f$ be a multifunction from or to a space $\mathbf{X}$ with the fixed-point property. In order to prove that $f$ is not continuous, it is sufficient to prove that $\mathbb{A}_{f}$ is continuous.

\section{The uniform fixed-point property}

As we will see later, most of (if not all) the known spaces having the fixed-point property even enjoy a uniform version of it, where a fixed-point can be continuously derived from the multifunction. We therefore define the uniform version of the fixed-point property, investigate its properties and relate it to the fixed-point property. We will see that it is indeed equivalent to the fixed-point property for many classes of spaces.

Remark 4.1. In [KW85], a uniform fixed-point property is considered, which corresponds to satisfying a form of the recursion theorem. However, that notion is very weak, for two reasons. First, it does not imply the fixed-point property considered here, because the fixed-point of $h$ : $\mathbf{X} \rightrightarrows \mathbf{X}$ realized by $H: \subseteq \mathcal{N} \rightarrow \mathcal{N}$ is some $p \in \mathcal{N}$ that can be, together with $H(p)$, outside the domain of $\delta_{X}$, therefore it may not correspond to an actual element of $\mathbf{X}$. The second reason why it is weak is that any representated space has an equivalent representation satisfying that form of the recursion theorem. Indeed, it is shown in [KW85] that this form of the recursion theorem is equivalent to precompleteness, and it is proved in [BGng] that every represented space has an equivalent representation that is precomplete.

The uniform fixed-point property that we define now is much stronger, in particular it implies the fixed-point property. It essentially says that every continuous multifunction $h: \mathbf{X} \rightrightarrows \mathbf{X}$ has a fixed-point, which can be continuously found from a name of $h$. What makes this notion different from the one considered in [KW85] is what happens when $h$ is partial. The definition may look somewhat arbitrary at first, but the next results show that it behaves very well. 
Definition 4.1. A represented space $\mathbf{X}$ has the (computable) uniform fixed-point property if given a name of a partial continuous function $H: \subseteq \mathcal{N} \rightarrow \mathcal{N}$ one can (computably) continuously produce some $p \in \operatorname{dom}\left(\delta_{X}\right)$ such that if $p \in \operatorname{dom}\left(\delta_{X} \circ H\right)$ then $\delta_{X}(p)=\delta_{X} \circ H(p)$.

Of course the computable version is stronger than the continuous one.

As we will see shortly, this notion is equivalent to two notions recently introduced. It coincides with the notion of a multi-retraceable represented space introduced by Brattka and Gherardi in [BG21]. It is also equivalent to the multifunction $x \mapsto\{y \in \mathbf{X}: y \neq x\}$ being effectively discontinuous as defined by Brattka in [Bra20]. We will give more details when needed, but these characterizations show that this notion is robust and meaningful.

Proposition 4.1. The uniform fixed-point property implies the fixed-point property.

Proof. Let $h: \mathbf{X} \rightrightarrows \mathbf{X}$ be continuous and let $H: \operatorname{dom}\left(\delta_{X}\right) \rightarrow \operatorname{dom}\left(\delta_{X}\right)$ be a continuous realizer of $h$. Observe that $\operatorname{dom}\left(\delta_{X} \circ H\right)=\operatorname{dom}\left(\delta_{X}\right)$. Applying the definition to $H$, there exists $p \in \operatorname{dom}\left(\delta_{X}\right)$ such that if $p \in \operatorname{dom}\left(\delta_{X} \circ H\right)$ then $\delta_{X}(p)=\delta_{X}(H(p))$. One indeed has $p \in \operatorname{dom}\left(\delta_{X} \circ H\right)$, so $\delta_{X}(p)=\delta_{X}(H(p))$. The point $x=\delta_{X}(p)$ is a fixed-point of $h$, as $x=\delta_{X}(H(p)) \in h(x)$.

Definition 4.1 may be difficult to work with. We give a simpler characterization which will be very useful in the sequel, and which shows that the computable uniform fixed-point property is equivalent to the notion of multi-retraceability introduced in [BG21], where many other characterizations are also proved.

Theorem 4.1 (Equivalence with the total extension property). Let $\left(\mathbf{X}, \delta_{X}\right)$ be a represented space. The following conditions are equivalent:

- $\mathbf{X}$ has the (computable) uniform fixed-point property,

- Every (computable) continuous function $f: \subseteq \mathcal{N} \rightarrow \mathbf{X}$ has a total (computable) continuous extension.

We need the next lemma.

Lemma 4.1. If every (computable) continuous function $f: \subseteq \mathcal{N} \rightarrow \mathbf{X}$ has a total (computable) continuous extension, then a name of a total extension can be (computably) continuously found from a name of $f$.

Proof. Let $U: \subseteq \mathcal{N} \times \mathcal{N} \rightarrow \mathbf{X}$ be a computable universal function. It has a total (computable) continuous extension $\bar{U}: \mathcal{N} \times \mathcal{N} \rightarrow \mathbf{X}$. Given a name of $f: \subseteq \mathcal{N} \rightarrow \mathbf{X}$, one can compute $p$ such that $f=U_{p}$. One can then (computably) continuously find a name of $\bar{U}_{p}$, which is a total extension of $f$.

Proof of Theorem 4.1. We prove the continuous version, which is effective and implies the computable version.

Assume that $\mathbf{X}$ has the uniform fixed-point property. Let $f: \subseteq \mathcal{N} \rightarrow \mathbf{X}$ be continuous. We define a total extension $g: \mathcal{N} \rightarrow \mathbf{X}$ of $f$ as follows. Let $F: \subseteq \mathcal{N} \rightarrow \mathcal{N}$ be a continuous realizer of $f$. Given $p \in \mathcal{N}$, define the constant function $H: \subseteq \mathcal{N} \rightarrow \mathcal{N}$ by $H(q)=F(p)$. Observe that if $p \notin \operatorname{dom}(f)$ then $H$ is nowhere defined. However in any case a name of $H$ can be uniformly computed from $p$ (and a name of $F$ ). Applying the uniform fixed-point property to $H$, one obtains $r \in \operatorname{dom}\left(\delta_{X}\right)$ such that if $r \in \operatorname{dom}(H)$ then $\delta_{X}(r)=\delta_{X}(H(r))$. Let then $g(p)=\delta_{X}(r)$. If $p \in \operatorname{dom}(f)$ then $H$ is defined everywhere so $r \in \operatorname{dom}(H)$, which implies that $\delta_{X}(r)=\delta_{X}(H(r))=f(p)$, so the function $g$ is indeed a total continuous extension of $f$. 
Conversely, assume that every partial function from $\mathcal{N}$ to $\mathbf{X}$ has a total extension. Let $H: \subseteq$ $\mathcal{N} \rightarrow \mathcal{N}$ be given. We apply the diagonal argument. Consider the universal computable function $U: \subseteq \mathcal{N} \times \mathcal{N} \rightarrow \mathcal{N}$. Define $F: \subseteq \mathcal{N} \rightarrow \mathcal{N}$ by $F(q)=H(U(q, q)) . F$ is a realizer of $f:=$ $\delta_{X} \circ F$, which has a total extension $g: \mathcal{N} \rightarrow \mathbf{X}$, with a continuous realizer $G: \mathcal{N} \rightarrow \mathcal{N}$. Let $a$ be such that $G(q)=U(a, q)$. Note that $G$, and therefore $a$, can be (computably) continuously found from $F$ by Lemma 4.1. We define $p=G(a)=U(a, a)$. First, $p \in \operatorname{dom}\left(\delta_{X}\right)$ because it is a name of $g(a)$. Second, if $p \in \operatorname{dom}(H)$ then $a \in \operatorname{dom}(F)$ so $\delta_{X}(p)=\delta_{X}(G(a))=\delta_{X}(F(a))=$ $\delta_{X}(H(p))$.

In particular, if $\mathbf{X}$ has the uniform fixed-point property, then it has an equivalent total representation, obtained by extending $\delta_{X}$. Note however that many spaces with a total representation do not have the uniform fixed-point property, for instance Polish spaces which are not singletons.

In the same way as retractions preserve the fixed-point property (Proposition 3.2), they preserve its uniform version.

Proposition 4.2. Let $\mathbf{X}$ have the (computable) uniform fixed-point property. A subspace $\mathbf{Y}$ of $\mathbf{X}$ has the (computable) uniform fixed-point property if and only if $\mathbf{Y}$ is a multivalued retract of $\mathbf{X}$.

Proof. If $\mathbf{Y}$ is a multivalued retract of $\mathbf{X}$, coming with $r, s$, then $\mathbf{Y}$ has the uniform fixed-point property. Indeed, if $f: \subseteq \mathcal{N} \rightarrow \mathbf{Y}$ is continuous then $s \circ f: \subseteq \mathcal{N} \rightarrow \mathbf{X}$ has a total continuous extension $f^{\prime}$, and $r \circ f^{\prime}$ is a total continuous extension of $f$.

Conversely, let $\mathbf{Y} \subseteq \mathbf{X}$ have the uniform fixed-point property. The restriction of $\delta_{X}$ to $\delta_{X}^{-1}(\mathbf{Y})$ is a partial continuous function from $\mathcal{N}$ to $\mathbf{Y}$ so it has a total continuous extension $f: \mathcal{N} \rightarrow \mathbf{Y}$. Let $s: \mathbf{Y} \rightarrow \mathbf{X}$ be the canonical injection and $r: \mathbf{X} \rightrightarrows \mathbf{Y}$ be defined by $r(x)=\left\{f(p): \delta_{X}(p)=x\right\}$. One has $r \circ s=\operatorname{id}_{\mathbf{Y}}$ so $r, s$ make $\mathbf{Y}$ a multivalued retract of $\mathbf{X}$.

It is proved in [BG21] that every represented space $\mathbf{X}$ with the uniform fixed-point property is a multivalued retract of a certain space $\overline{\mathbf{X}}$ called the completion of $\mathbf{X}$ (which we do not need to define here). The previous result implies that when $\mathbf{X}$ is countably-based, on can use $\mathcal{P}(\omega)$ instead of $\overline{\mathbf{X}}$.

Corollary 4.1. A countably-based $T_{0}$-space has the (computable) uniform fixed-point property if and only if it is a (computable) multivalued retract of $\mathcal{P}(\omega)$.

Proof. The countably-based $T_{0}$-spaces are the subspaces of $\mathcal{P}(\omega)$, which satisfies the computable uniform fixed-point property, so we can apply Proposition 4.2.

Note that the notion of multivalued retract is particularly useful here as it is not possible in general to make $\mathbf{X}$ a single-valued retract of $\mathcal{P}(\omega)$. Indeed, every single-valued retract of $\mathcal{P}(\omega)$ has a greatest element, which is the image of $\omega$ under the retraction. However, the space of partial functions from $\mathbb{N}$ to $\mathbb{N}$, which has the uniform fixed-point property, has no greatest element.

Proposition 4.3. A represented space $\mathbf{X}$ has the (computable) uniform fixed-point property if and only if $\mathcal{C}(\mathcal{N}, \mathbf{X})$ has.

Proof. The argument is the same for the continuous and computable cases, we formulate the continuous case only.

Assume that $\mathbf{X}$ has the total extension property. A partial continuous function $f: \subseteq \mathcal{N} \rightarrow$ $\mathcal{C}(\mathcal{N}, \mathbf{X})$ can be translated into a partial continuous function $g: \subseteq \mathcal{N} \rightarrow \mathbf{X}$ by currying and 
pairing. If $g^{\prime}$ is a total continuous extension of $g$, then it can be translated back to a total continuous function $f^{\prime}: \mathcal{N} \rightarrow \mathcal{C}(\mathcal{N}, \mathbf{X})$, which is a total extension of $f$.

Now assume that $\mathcal{C}(\mathcal{N}, \mathbf{X})$ has the total extension property. A partial continuous function $g: \subseteq \mathcal{N} \rightarrow \mathbf{X}$ can be translated into a partial continuous function $f: \subseteq \mathcal{N} \rightarrow \mathcal{C}(\mathcal{N}, \mathbf{X})$ whose values are constant functions: $f(p)(q)=g(p)$. If $f^{\prime}$ is a total continuous extension of $f$ then $g^{\prime}(p)=f^{\prime}(p)(p)$ is a total continuous extension of $g$.

We do not know whether a similar result holds for the non-uniform fixed-point property.

We now show a result suggesting that for any concrete space, the uniform fixed-point property is actually equivalent to the fixed-point property. This result follows from the results in [Bra20], but we also include a self-contained proof.

Theorem 4.2. Let $\mathbf{X}$ be a represented space. Assuming the Axiom of Determinacy, the following conditions are equivalent:

- $\mathbf{X}$ has the fixed-point property,

- $\mathbf{X}$ has the uniform fixed-point property.

For a concrete represented space $\mathbf{X}$, the full Axiom of Determinacy is usually not needed. If $\operatorname{dom}\left(\delta_{X}\right)$ and $\left\{p, q \in \mathcal{N}: \delta_{X}(p)=\delta_{X}(q)\right\}$ are Borel, then the result holds without any assumption. If those sets are boolean combinations of analytic sets, then $\boldsymbol{\Sigma}_{1}^{1}$-determinacy is sufficient. We will see classes of spaces where those sets are not necessarily Borel, but the equivalence holds without any assumption (Theorem 6.1). As explained in [Kec95] (Section 26.B there), there is strong evidence that determinacy for "definable" sets is valid, so one can expect that the equivalence holds for any "definable" represented space. In the sequel, we will give specific proofs without assuming the Axiom of Determinacy, for some classes of spaces.

We do not do any claim about the validity of the Axiom of Determinacy, which may be considered as a very strong assumption. However, this result suggests that for each particular space, it may be possible to prove either that $\mathbf{X}$ has the uniform fixed-point property, or that $\mathbf{X}$ does not have the fixed-point property.

Proof. Let us first show how it relates follows from recent results appearing in [Bra20]. Let $f$ : $\mathbf{X} \rightrightarrows \mathbf{X}$ be defined by $f(x)=\{y \in \mathbf{X}: x \neq y\}$. $\mathbf{X}$ has the fixed-point property iff $f$ is not continuous. $\mathbf{X}$ has the uniform fixed-point property iff $f$ is effectively discontinuous as defined in [Bra20]. It is proved there that under the Axiom of Determinacy, every multifunction between represented spaces is either continuous or effectively discontinuous.

We now include a proof. Consider the following game between Player I and II, who alternatively play an element in $\mathbb{N}_{\#}=\mathbb{N} \cup\{\#\}$, starting with Player I. If a sequence $s \in \mathcal{N}_{\#}:=\left(\mathbb{N}_{\#}\right)^{\mathbb{N}}$ contains infinitely many natural numbers, then we say that $s$ is valid and define $p_{s} \in \mathcal{N}$ as the sequence obtained by removing the occurrences of $\#$ in $s$. The goal of introducing the symbol \# is that all partial continuous functions from $\mathcal{N}$ to $\mathcal{N}$ can be turned into strategies for the players. This idea is called the precompletion of a representation, see [KW85, BG21] for instance. Using the precompletion to express continuous multifunctions in terms of games has been done by Nobrega and Pauly in [NP19].

Let $D=\left\{s \in \mathcal{N}_{\#}: s\right.$ is valid and $\left.p_{s} \in \operatorname{dom}\left(\delta_{X}\right)\right\}$. Let $s, t$ be the sequences played by Players I and II respectively. We say that

- Player II wins if $s \in D$ implies $\left(t \in D\right.$ and $\left.\delta_{X}\left(p_{t}\right) \neq \delta_{X}\left(p_{s}\right)\right)$,

- Player I wins if Player II does not win, i.e. $s \in D$ and $\left(t \in D\right.$ implies $\left.\delta_{X}\left(p_{t}\right)=\delta_{X}\left(p_{s}\right)\right)$. 
Player II has a winning strategy if and only if $D$ admits a continuous fixed-point free multifunction $h: \mathbf{X} \rightrightarrows \mathbf{X}$.

Player I has a winning strategy if and only if there exists a total continuous function $f$ : $\mathcal{N}_{\#} \rightarrow \mathbf{X}$ such that if $t \in D$ then $f(t)=\delta_{X}\left(p_{t}\right)$.

Assume the Axiom of Determinacy, the game is determined, i.e. one of the Players has a strategy. If Player II has a winning strategy then $\mathbf{X}$ does not have the fixed-point property. If Player I has a winning strategy, then every partial continuous function $g: \subseteq \mathcal{N} \rightarrow \mathbf{X}$ has a total extension hence $\mathbf{X}$ has the uniform fixed-point property. Indeed, let $G: \subseteq \mathcal{N} \rightarrow \mathcal{N}$ be a continuous realizer of $g$, and let $G^{\prime}: \mathcal{N} \rightarrow \mathcal{N}_{\#}$ be a total continuous function such that if $p \in \operatorname{dom}(g)$ and $t=G^{\prime}(p)$, then $t \in D$ and $\delta_{X}\left(p_{t}\right)=g(p)$. The function $f \circ G^{\prime}$ is a total extension of $g$.

Theorem 4.3. Assuming the Axiom of Choice, there exists a represented space satisfying the fixed-point property but not the uniform fixed-point property.

Proof. Let $X=\{0,1\}$ and $\emptyset \neq A \subsetneq \mathcal{N}$. Let $\delta_{A}: \mathcal{N} \rightarrow X$ be the characteristic function of $A$, defined by $\delta_{A}(p)=1 \Longleftrightarrow p \in A$. We consider the represented space $\mathbf{X}_{A}=\left(X, \delta_{A}\right)$. We are going to build $A$ so that $\mathbf{X}_{A}$ satisfies the statement of the theorem. Let us first see how it translates in terms of the properties of $A$.

First, $\mathbf{X}_{A}$ has the fixed-point property if and only if $A$ is not continuously reducible to $A^{c}$. Indeed, the existence of a fixed-point free continuous multifunction boils down to the exitence of a continuous realizer $H: \mathcal{N} \rightrightarrows \mathcal{N}$ such that $p \in A \Longleftrightarrow H(p) \notin A$ for all $p \in \mathcal{N}$, i.e. a continuous reduction from $A$ to $A^{c}$. We will build $A$ so that $A$ is not reducible to $A^{c}$, so that $\mathbf{X}_{A}$ has the fixed-point property.

We now define a partial continuous function $g: \subseteq \mathcal{N} \rightarrow \mathbf{X}$ and we will build $A$ so that $g$ has no total continuous extension, implying that $\mathbf{X}_{A}$ does not have the uniform fixed-point property. Let $G: \subseteq \mathcal{N} \rightarrow \mathcal{N}$ be the continuous partial function that takes a non-null $p \in \mathcal{N}$, removes all the leading 0's and decrements the first non-null element: more precisely, if $n \in \mathbb{N}$ is minimal such that $p(n) \neq 0$, then $G(p)=(p(n)-1, p(n+1), p(n+2), \ldots)$. The function $G$ is a continuous realizer of $g:=\delta_{A} \circ G: \subseteq \mathcal{N} \rightarrow \mathbf{X}$.

The important property of $G$ is that it has no total continuous extension, and moreover for every continuous function $F: \mathcal{N} \rightarrow \mathcal{N}$, the set $\{G(q): q \in \mathcal{N}, F(q) \neq G(q)\}$ has cardinality . Indeed, let $s=F\left(0^{\omega}\right)$, let $n$ be such that every element of $F\left(\left[0^{n}\right]\right)$ starts with $s(0)$ and let $k=$ $s(0)+2$. For each $q$ starting with $0^{n} k, F(q)$ starts with $s(0)$ while $G(q)$ starts with $s(0)+1$, so $F(q) \neq G(q)$. All the elements of $\mathcal{N}$ starting with $s(0)+1$ can be obtained as $G(q) \neq F(q)$.

We follow the standard way of building a set by transfinite induction over the ordinals $\alpha<\mathfrak{c}$, used for instance in the construction of a Bernstein set [Oxt13].

Let $\left(F_{\alpha}\right)_{\alpha<\mathfrak{c}}$ be an enumeration of all the continuous functions from $\mathcal{N}$ to $\mathcal{N}$, and $\left(p_{\alpha}\right)_{\alpha<\mathfrak{c}}$ an enumeration of the elements of $\mathcal{N}$. We build by induction on $\alpha<\mathfrak{c}$ two growing families of disjoint sets $A_{\alpha}, B_{\alpha}$ of cardinalities $<\mathfrak{c}$, and define $A=\bigcup_{\alpha<\mathfrak{c}} A_{\alpha}$.

We start with $A_{0}=B_{0}=\emptyset$. For a limit ordinal $\alpha$, we let $A_{\alpha}=\bigcup_{\beta<\alpha} A_{\beta}$ and $B_{\alpha}=\bigcup_{\beta<\alpha} B_{\beta}$. We now build $A_{\alpha+1}, B_{\alpha+1}$ from $A_{\alpha}, B_{\alpha}$, adding a finite number of elements to them.

In order to prevent $F_{\alpha}$ from being a reduction from $A$ to $A^{c}$, let $p$ be the first element of $\mathcal{N}$ which is not in $A_{\alpha} \cup B_{\alpha}$. If $F_{\alpha}(p) \in A_{\alpha}$ then we add $p$ to $A_{\alpha+1}$; if $F_{\alpha}(p) \in B_{\alpha}$ then we add $p$ to $B_{\alpha+1}$; otherwise we add both $p$ and $F_{\alpha}(p)$ to $A_{\alpha+1}$.

In order to prevent $F_{\alpha}$ from realizing an extension of $g$, let $q$ be the first element of $\mathcal{N}$ such that $G(q)$ is not in $A_{\alpha} \cup B_{\alpha} \cup\left\{p, F_{\alpha}(p)\right\}$, and such that $F_{\alpha}(q) \neq G(q)$. If $F_{\alpha}(q) \in A_{\alpha}$ then we add $G(q)$ to $B_{\alpha+1}$; if $F_{\alpha}(q) \in B_{\alpha}$ then we add $G(q)$ to $A_{\alpha}+1$; otherwise we add $G(q)$ to $A_{\alpha+1}$ and $F_{\alpha}(q)$ to $B_{\alpha+1}$. 
For each $\alpha<\mathfrak{c}$, there is some $p \in \mathcal{N}$ such that $p \in A \Longleftrightarrow F_{\alpha}(p) \in A$, so $A$ is not reducible to its complement and $\mathbf{X}$ has the fixed-point property.

For each $\alpha<\mathfrak{c}$, there is some $q \in \mathcal{N}$ such that $F_{\alpha}(q) \in A \Longleftrightarrow G(q) \notin A$, so $\delta_{A} \circ F_{\alpha}$ does not extend $g$. Therefore, $\mathbf{X}$ does not have the uniform fixed-point property.

We leave the following question open: is there an admissibly represented space satisfying the fixed-point property, but not the uniform one? We will see later that it cannot be a countablybased $T_{0}$-space with the standard representation.

\subsection{Complexity of equality}

We now show that for a subspace $\mathbf{Y}$ of a given space $\mathbf{X}$, there is a loose relationship between the symbolic complexity of $\mathbf{Y}$ and whether $\mathbf{Y}$ has the (uniform) fixed-point property: if $\mathbf{Y} \subseteq \mathbf{X}$ has the uniform fixed-point property, then $\mathbf{Y}$ is no more complex than equality on $\mathbf{X}$. The descriptive complexity of equality also plays an important role in [dBSS16].

For instance, for countably-based $T_{0}$-spaces, i.e. subspaces of $\mathcal{P}(\omega)$, having the uniform fixed-point property implies being ${\underset{\sim}{2}}_{2}^{0}$, i.e. quasiPolish.

As a result, if $\mathbf{Y}$ is more complex than equality then it does not have the uniform fixed-point property. Assuming the Axiom of Determinacy it does not have the fixed-point property either by Theorem 4.2. We can drop the determinacy assumption if we require that $\mathbf{Y}$ is not only more complex than equality, but hard for the corresponding complexity level.

Theorem 4.4. Let $\Gamma$ be $\underset{\sim}{\boldsymbol{\Sigma}_{\alpha}^{0}},{\underset{\sim}{\mathbf{D}}}_{\beta}\left(\boldsymbol{\Sigma}_{\alpha}^{0}\right),{\underset{\sim}{\boldsymbol{\Sigma}}}_{\alpha}^{1}$ or their dual classes ${\underset{\sim}{\boldsymbol{\Pi}}}_{\alpha}^{0}, \check{\mathbf{D}}_{\beta}\left({\underset{\sim}{\boldsymbol{\Sigma}}}_{\alpha}^{0}\right),{\underset{\sim}{\boldsymbol{\sim}}}_{\alpha}^{1}$, where $\alpha, \beta$ are countable ordinals. If equality on $\mathbf{X}$ has complexity $[\Gamma](\mathbf{X} \times \mathbf{X})$, then for $A \subseteq \mathbf{X}$ :

- If $A$ has the fixed-point property then $A$ is not $\check{\Gamma}$-hard,

- If $A$ has the uniform fixed-point property then $A \in[\Gamma](\mathbf{X})$.

We will use the fact that these descriptive complexity classes enjoy the following standard properties:

Properties 4.1. - $\Gamma$ has a universal set $U \in \Gamma(\mathcal{N} \times \mathcal{N})$, which means that $\Gamma(\mathcal{N})=\left\{U_{p}: p \in\right.$ $\mathcal{N}\}$ where $U_{p}=\{q \in \mathcal{N}:(p, q) \in U\}$,

- $\Gamma$ is closed under taking continuous preimages, moreover for every continuous function $f$ : $\mathcal{N} \rightarrow \mathcal{N}$, there exists a continuous function $g: \mathcal{N} \rightarrow \mathcal{N}$ such that $f^{-1}\left(U_{p}\right)=U_{g(p)}$.

These properties have the following interesting consequence: not only $\check{\Gamma}$-hard sets differ from each $\Gamma$-set, but a witness of this difference can be continuously found from any $\Gamma$-set, as the next results show.

Lemma 4.2. Let $\Gamma$ satisfy Properties 4.1 and $A \subseteq \mathcal{N}$ be $\check{\Gamma}$-hard. There exists a continuous function $f: \mathcal{N} \rightarrow \mathcal{N}$ such that $f(p) \in U_{p} \triangle A$ for all $p \in \mathcal{N}$.

Proof. Consider the diagonal $D_{U}:=\left\{p \in \mathcal{N}: p \in U_{p}\right\}$, which belongs to $\Gamma(\mathcal{N})$. As $A$ is $\check{\Gamma}$-hard, there exists a continuous function $g: \mathcal{N} \rightarrow \mathcal{N}$ such that $D_{u}^{c}=g^{-1}(A)$. Let $h: \mathcal{N} \rightarrow \mathcal{N}$ be a continuous function such that $U_{h(p)}=g^{-1}\left(U_{p}\right)$, and let $f=g \circ h$. By definition of the diagonal, one has $h(p) \in U_{h(p)} \triangle D_{U}^{c}=g^{-1}\left(U_{p} \triangle A\right)$, so $f(p)=g(h(p)) \in U_{p} \triangle A$.

Let $\left(\mathbf{X}, \delta_{X}\right)$ be a represented space. The class $[\Gamma](\mathbf{X})$ has a natural representation: a name of $S \in[\Gamma](\mathbf{X})$ is any name of a set $T \in \Gamma(\mathcal{N})$ such that $\delta_{X}^{-1}(S)=T \cap \operatorname{dom}\left(\delta_{X}\right)$.

Corollary 4.2. Let $\Gamma$ satisfy Properties 4.1 and $A \subseteq \mathbf{X}$ be $\check{\Gamma}$-hard. The multifunction $f$ : $[\Gamma](\mathbf{X}) \rightrightarrows \mathbf{X}$ defined by $f(S)=A \triangle S$ is continuous. 
Proof. As $A$ is $\check{\Gamma}$-hard, there exists a continuous function $g: \mathcal{N} \rightarrow \operatorname{dom}\left(\delta_{X}\right)$ such that $D_{U}^{c}=$ $g^{-1}\left(\delta_{X}^{-1}(A)\right)$. As in the proof of Lemma 4.2 we can define a continuous function $f_{0}: \mathcal{N} \rightarrow$ $\operatorname{dom}\left(\delta_{X}\right)$ such that $f_{0}(p) \in U_{p} \triangle \delta_{\mathbf{X}}^{-1}(A)$ for all $p \in \mathcal{N}$. The function $f_{0}$ is precisely a continuous realizer of $f$.

Proof of Theorem 4.4. We assume that $A$ is $\check{\Gamma}$-hard and build a fixed-point free continuous multifunction $h: A \rightrightarrows A$. As equality belongs to $[\Gamma](\mathbf{X} \times \mathbf{X})$, the function $s: \mathbf{X} \rightarrow[\Gamma](\mathbf{X})$ sending $x$ to $\{x\}$ is continusouly realizable. Corollary 4.2 implies that $f:[\Gamma](\mathbf{X}) \rightrightarrows \mathbf{X}$ sending $S$ to $A \triangle S$ is continuous. We simply compose the two functions and define $h=f \circ s$. For all $x \in \mathbf{X}$, one has $h(x) \subseteq A \triangle\{x\}$, in particular $h(x) \subseteq A \backslash\{x\}$ when $x \in A$. $h: A \rightrightarrows A$ is continuous and fixed-point free.

Now let $A$ have the uniform fixed-point property. The restriction of $\delta_{X}$ to the pre-image of $A$ has a total extension $g: \mathcal{N} \rightarrow A$. One has $\delta_{X}^{-1}(A)=\left\{p \in \operatorname{dom}\left(\delta_{X}\right): \delta_{X}(p)=g(p)\right\}$. As equality belongs to $[\Gamma]$, so does $A$.

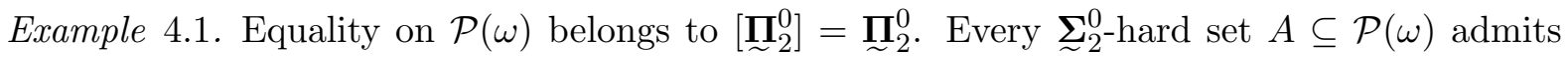
a fixed-point free continuous multifunction $h: A \rightrightarrows A$. If a set $A \subseteq \mathcal{P}(\omega)$ has the uniform fixed-point property then it is $\boldsymbol{\Pi}_{2}^{0}$, i.e. it is quasiPolish. We will see soon that the fixed-point property already implies $A \in \prod_{2}^{0}$, but the proof is much more involved (Theorem 5.1).

Example 4.2. Equality on $\mathcal{O}(\mathcal{N})$ belongs to $\left[\boldsymbol{\Pi}_{1}^{1}\right]$. Therefore, every $\boldsymbol{\Sigma}_{1}^{1}$-hard set $A \subseteq \mathcal{O}(\mathcal{N})$ admits a fixed-point free continuous multifunction $h: A \rightrightarrows A$. Every subset of $\mathcal{O}(\mathcal{N})$ satisfying the uniform fixed-point property is in $\left[{\underset{\sim}{1}}_{1}^{1}\right]$.

\section{Countably-based spaces}

We show that for countably-based $T_{0}$-spaces, the fixed-point property is equivalent to its uniform version: for such spaces Theorem 4.2 can be proved without assuming the Axiom of Determinacy. We moreover give several characterizations of the countably-based spaces with the fixed-point property. Interestingly, they are exactly the pointed $\omega$-continuous dcpos endowed with the Scott topology, a particularly important class of spaces from domain theory.

Observe that one probably cannot hope for a characterization of the countably-based $T_{0^{-}}$ spaces having the more traditional fixed-point property for single-valued functions. For instance, a consequence of our results is that the finite $T_{0}$-spaces having the fixed-point property for multifunctions are exactly the ones with a least element, while characterizing the finite $T_{0}$-spaces having the fixed-point property for functions is a difficult unsolved problem (it is equivalent to the fixed-point property for finite partial orders which is an open problem, see Section 7 in [Sch12]).

We now state and prove the characterization of countably-based $T_{0}$-spaces with the (uniform) fixed-point property.

Theorem 5.1. Let $X$ be a countably-based $T_{0}$-space. The following statements are equivalent:

1. $X$ has the fixed-point property,

2. $X$ has the uniform fixed-point property,

3. $X$ is a multivalued retract of $\mathcal{P}(\omega)$,

4. $X$ is a quasiPolish c-space with a least element,

5. $X$ is homeomorphic to a pointed $\omega$-continuous dcpo endowed with the Scott topology. 
Moreover, in 5., the partial order is the specialization order on $X$. We cut the proof into several intermediate results.

The proof is rather involved. We cut it into several intermediate results.

Proposition 5.1. Let $\mathbf{X}$ be a countably-based $T_{0}$-space. If $\mathbf{X}$ has the fixed-point property, then $\mathbf{X}$ is a c-space.

Proof. Assume that some $y_{0} \in \mathbf{X}$ is not in the closure of $\downarrow_{t} y_{0}$. We build a continuous fixed-point free multifunction $h: \mathbf{X} \rightrightarrows \mathbf{X}$. We assume that $\mathbf{X} \subseteq \mathcal{P}(\omega)$.

There exists a finite set $F \subseteq y_{0}$ such that there is no $x \in[F) \cap \mathbf{X}$ with $x \ll_{t} y_{0}$. In other words, for each $x \in[F] \cap \mathbf{X}, y_{0}$ is not in the interior of $\uparrow x$.

Given $x \in \mathbf{X}$, we produce some $z \in \mathbf{X}$ different from $x$. Start producing $y_{0}$. If we eventually see that $x \in[F]$, let $G$ be the finite subset of $y_{0}$ that we have enumerated so far. We know that $y_{0}$ is not in the interior of $\uparrow x$, in particular $[G] \cap \mathbf{X}$ is not contained in $\uparrow x$, so there exists $n \in x$ and $z \in[G] \cap \mathbf{X}$ such that $n \notin z$ (implying $x \not \leq z$ ). We can wait to see such an $n \in x$ and $z$. We then enumerate $z$.

Remark 5.1. This result does not hold outside countably-based spaces. We will see that the space $\mathcal{O}(\mathcal{N})$ has the uniform fixed-point property because $\mathcal{N}$ is countably-based (Theorem 6.1). However it is not a c-space, because for $x, y \in \mathcal{O}(\mathcal{N})$, one has $x \ll_{t} y$ if and only if $x=\emptyset$.

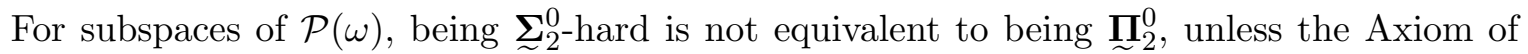
Determinacy is assumed. However, among c-spaces, the equivalence holds without any assumption. We see here why the Axiom of Determinacy is not needed to prove the equivalence between the fixed-point property and its uniform version for countably-based $T_{0}$-spaces.

Proposition 5.2. Let $\mathbf{X}$ be a countably-based $T_{0}$-space and consider the following conditions:

1. $\mathbf{X}$ is quasiPolish (i.e. embeds as a $\mathbf{\Pi}_{2}^{0}$-subset of $\mathcal{P}(\omega)$ ),

2. $\mathbf{X}$ is not $\Sigma_{2}^{0}$-hard, as a subset of $\mathcal{P}(\omega)$,

3. $\mathbf{X}$ is sober.

One has $1 . \Rightarrow 2 . \Rightarrow 3$. , and they are all equivalent when $\mathbf{X}$ is a c-space.

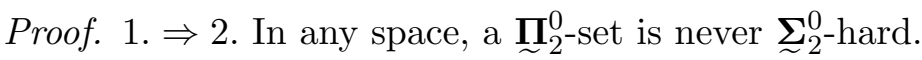

2. $\Rightarrow 3$. If $\mathbf{X}$ is not sober, then there exists a sequence $x_{i} \in \mathbf{X}$ converging to $x \in \mathcal{P}(\omega) \backslash \mathbf{X}$, such that $x_{i} \leq x$ (see Proposition 2.2). One can easily build a continuous reduction from the set of binary sequences with finitely many 1 's to $\mathbf{X}$. Let $n_{0}, n_{1}, \ldots$ be an enumeration of $x$. Given a binary sequence $s$, we enumerate some element of $\mathcal{P}(\omega)$. We start enumerating $x_{0}$, and at any stage we are enumerating some $x_{i}$. Each time we see a new occurrence of 1 in $s$, we update the number $k$ of 1 's seen so far in $s$. If $F$ is the finite subset of $x$ we have enumerated so far, then there exists $i$ such that $x_{i}$ contains both $F$ and $n_{k}$. We then enumerate $x_{i}$. If $s$ contains finitely many 1's then we eventually stabilize, enumerating some $x_{i} \in \mathbf{X}$. If $s$ contains infinitely many 1 's then in the limit we enumerate $x \notin \mathbf{X}$.

3 . $\Rightarrow 1$. If $\mathbf{X}$ is a c-space then it is locally compact. Every countably-based locally compact sober space is quasiPolish by Theorem 44 in [dB13].

Proposition 5.3. Every quasiPolish c-space with a least element is a multivalued retract of $\mathcal{P}(\omega)$. 
Proof. We assume that $\mathbf{X} \subseteq \mathcal{P}(\omega)$ and build a multivalued retraction $r: \mathcal{P}(\omega) \rightrightarrows \mathbf{X}$. Note that $\mathbf{X}$ is a $\mathbb{\sim}_{2}^{0}$-subset of $\mathcal{P}(\omega)$. The specialization order is simply the restriction to $\mathbf{X}$ of the subset order on $\mathcal{P}(\omega)$. Given $x \in \mathcal{P}(\omega)$, we show how to build $y=r(x) \in \mathbf{X}$ such that $y=x$ if $x \in \mathbf{X}$. We start enumerating the least element $x_{0} \in \mathbf{X}$.

At each stage we are enumerating some $y \in \mathbf{X}$ with $y \leq x$, starting with $y=x_{0}$. If $x \in \mathbf{X}$ then we may change our mind infinitely often and converge to $x$, otherwise we stabilize on

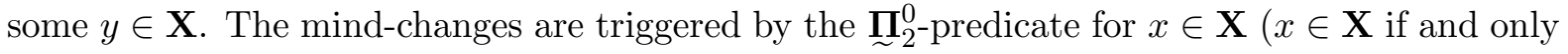
if infinitely many mind-changes are triggered).

When a mind-change is triggered, let $F_{0}$ be the finite set we have enumerated so far in $y \in \mathbf{X}$ and $F_{1}$ be the finite set that has been enumerated so far in $x$. Let $F=F_{0} \cup F_{1}$, which is contained in $x$, because $y \leq x$. We look for some $z \in \mathbf{X} \cap[F]$ such that $z \ll_{t} x$. If we never find such a $z$, then we keep enumerating the current $y$ and ignore the subsequent mind-change triggers. If we eventually find such a $z$, then we switch from $y$ to $z$ and proceed.

If $x \in \mathbf{X}$ then each triggered mind-change leads to finding some $z \in \mathbf{X}$ such that $F \subseteq z \ll_{t} x$. As a result, in the limit we enumerate all the elements of $x$ and only elements of $x$, so we are enumerating $x$. If $x \notin \mathbf{X}$ then after some finite time, no mind-change will ever occur and we enumerate some $y \in \mathbf{X}$.

We can now combine these results to prove the equivalence between the first four conditions in Theorem 5.1 .

Proof of Theorem 5.1. 4. $\Rightarrow$ 3. is Proposition 5.3. 3. $\Leftrightarrow 2$. is Corollary 4.1. 2. $\Rightarrow 1$. is Proposition 4.1. 1. $\Rightarrow 4$. If $\mathbf{X}$ has the fixed-point property, then $\mathbf{X}$ has a least element (Proposition

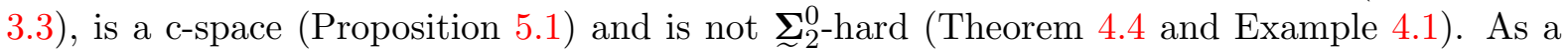
result, it is quasiPolish by Proposition 5.2. 4. $\Leftrightarrow 5$. Theorem 2.1 states that the sober c-spaces are exactly the continuous dcpos with their Scott topology. We also know by Proposition 5.2 that the sober c-spaces are exactly the quasiPolish c-spaces. Moreover, the Scott topology on a continuous dcpo is countably-based if and only if the dcpo is $\omega$-continuous.

\section{Classes of spaces}

We now study other classes of topological spaces, giving for each class a characterization of the spaces with the (uniform) fixed-point property.

\subsection{Spaces of open sets}

We study the space of open subsets $\mathcal{O}(\mathbf{X})$ of an admissibly represented space $\mathbf{X}$. This space has an admissible representation inducing the Scott topology [Sch15]. We give a very simple characterization the spaces $\mathbf{X}$ for which $\mathcal{O}(\mathbf{X})$ satisfies the fixed-point property, and show that for $\mathcal{O}(\mathbf{X})$ the fixed-point property is equivalent to its uniform version, giving another instantiation of Theorem 4.2 without the Axiom of Determinacy.

Observe that Kleene's fixed-point theorem implies that every single-valued continuous function $f: \mathcal{O}(\mathbf{X}) \rightarrow \mathcal{O}(\mathbf{X})$ has a fixed-point. However, continuous multifunctions may have no fixed-point.

Theorem 6.1. Let $\mathbf{X}$ be admissibly represented. The following conditions are equivalent:

- $\mathbf{X}$ is countably-based,

- $\mathcal{O}(\mathbf{X})$ has the uniform fixed-point property, 
- $\mathcal{O}(\mathbf{X})$ has the fixed-point property.

Proof. We assume that $\mathbf{X}$ is not countably-based and build a fixed-point free continuous multifunction $h: \mathcal{O}(\mathbf{X}) \rightrightarrows \mathcal{O}(\mathbf{X})$. The proof shares some similarity with the proof of Theorem 4.5 in [CH20]. We can assume w.l.o.g. that the sets $\delta_{X}([\sigma])$ are upwards closed for the specialization order on $\mathbf{X}$ (Lemma 4.6 in [CH20]). Let $\left(C_{i}\right)_{i \in \mathbb{N}}$ be an enumeration of the finite unions of cylinders in $\mathcal{N}$. The interiors of the sets $\delta_{X}\left(C_{i}\right)$ form a countable family of open sets, so this family is not a basis. Therefore, there exists an open set $O \in \mathcal{O}(\mathbf{X})$ and a point $x \in O$ such that for each $i \in \mathbb{N}$, if $\delta_{X}\left(C_{i}\right)$ is contained in $O$ then $x$ does not belong to the interior of $\delta_{X}\left(C_{i}\right)$.

We build a continuous fixed-point free multifunction $h: \mathcal{O}(\mathbf{X}) \rightrightarrows \mathcal{O}(\mathbf{X})$, i.e., given a name of $U \in \mathcal{O}(\mathbf{X})$, we produce a name of some $V \in \mathcal{O}(\mathbf{X})$ such that $V \neq U$. We start producing a name of $O$, which is a list of cylinders, and test whether $x \in U$.

If we eventually detect that $x \in U$, then let $C_{i}$ be the finite union of prefixes of names enumerated so far in the name of $O$. As $\delta_{X}\left(C_{i}\right)$ is contained in $O, x$ does not belong to its interior. In particular, $\delta_{X}\left(C_{i}\right)$ does not contain $U$, so reading the name of $U$, we can eventually find some $y \in U \backslash \delta_{X}\left(C_{i}\right)$ (It might not be clear that we can find such a $y$, so let us clarify this point. First observe that we do not require the procedure to be computable, but continuous only. We can imagine that before the pseudo-algorithm starts, we have fixed an infinite list of the pairs $(i, j)$ such that $\delta_{X}\left(C_{i}\right)$ does not contain $\delta_{X}\left(C_{j}\right)$, and for each such pair, we have chosen a point $y_{i, j}$ in $\delta_{X}\left(C_{j}\right) \backslash \delta_{X}\left(C_{i}\right)$; at the stage of pseudo-algorithm when we know that $\delta_{X}\left(C_{i}\right)$ does not contain $U$, we just have to find a pair $(i, j)$ in this list, where $C_{j}$ is some finite approximation of $U$, and then let $\left.y=y_{i, j}\right)$. We then let $V=\mathbf{X} \backslash \operatorname{cl}(\{y\})$ and extend the current name accordingly. It is possible because $\delta_{X}\left(C_{i}\right)$ is contained in $V$. Indeed $\delta_{X}\left(C_{i}\right)$ is upwards closed and does not contain $y$, so it is disjoint from $\operatorname{cl}(\{y\})$.

If $x \notin U$, then we continue producing a name of $O$ forever so $V=O$.

In the first case, one has $y \in U$ but $y \notin V$. In the second case, one has $x \notin U$ but $x \in V$. In both cases, one has $V \neq U$.

We now show that if $\mathbf{X}$ is countably-based then $\mathcal{O}(\mathbf{X})$ has the uniform fixed-point property. Let $\left(B_{i}\right)_{i \in \mathbb{N}}$ be a countable basis of $\mathbf{X}$. An open set $U \in \mathcal{O}(\mathbf{X})$ can be equivalently represented as a union of basic open sets, i.e. as a set $E \in \mathcal{P}(\omega)$ such that $U=\bigcup_{i \in E} B_{i}$. It means that the function $r: \mathcal{P}(\omega) \rightarrow \mathcal{O}(\mathbf{X})$ defined by $r(E)=\bigcup_{i \in E} B_{i}$ is continuously realizable and the multifunction $s: \mathcal{O}(\mathbf{X}) \rightrightarrows \mathcal{P}(\omega)$ defined by $s(U)=r^{-1}(U)$ is continuously realizable. Note that the pair $(r, s)$ is like a retraction-section pair, because $r \circ s=\mathrm{id}$, but it is not a multivalued retraction as in Definition 3.1, because $s$ is multi-valued. However this version of retraction also preserves the uniform fixed-point property, as we now show.

Given a partial continuous function $f: \subseteq \mathcal{N} \rightarrow \mathcal{O}(\mathbf{X})$, the multifunction $s \circ f: \subseteq \mathcal{N} \rightrightarrows \mathcal{P}(\omega)$ is continuous so by Proposition 2.1 it has a continuous single-valued selection $g: \subseteq \mathcal{N} \rightarrow \mathcal{P}(\omega)$, with $g(p) \in s \circ f(p)$. As $\mathcal{P}(\omega)$ satisfies the uniform fixed-point property, $g$ has a total continuous extension $h: \mathcal{N} \rightarrow \mathcal{P}(\omega)$. The continuous function $r \circ h: \mathcal{N} \rightarrow \mathcal{O}(\mathbf{X})$ is a total extension of $f$, because if $p \in \operatorname{dom}(f)$ then $p \in \operatorname{dom}(g)$ so $h(p)=g(p) \in s(f(p))$, hence $r \circ h(p) \in r(s(f(p))=$ $\{f(p)\}$.

One can ask for which $\mathbf{X}$ the space $\mathcal{O}(\mathcal{O}(\mathbf{X}))$ has the fixed-point property, i.e. for which $\mathbf{X}$ the space $\mathcal{O}(\mathbf{X})$ is countably-based. This question is well-understood when $\mathbf{X}$ is Hausdorff.

Theorem 6.2 (Schröder, Theorem 7.3 in [Sch04]). Let $\mathbf{X}$ be a Hausdorff admissibly represented space. The following conditions are equivalent:

- $\mathcal{O}(\mathbf{X})$ is countably-based,

- $\mathcal{O}(\mathbf{X})$ is quasiPolish, 
- $\mathbf{X}$ is coPolish, i.e. is the inductive limit of a growing sequence of compact metrizable spaces.

In particular, when $\mathbf{X}$ is Hausdorff and countably-based, being coPolish is equivalent to being locally compact.

We also give an answer when $\mathbf{X}$ is countably-based but not necessarily Hausdorff. The proof uses preceding results about the fixed-point property. We do not know whether a more direct proof can be found.

Definition 6.1. A topological space $\mathbf{X}$ is core-compact if its lattice of open sets $(\mathcal{O}(\mathbf{X}), \subseteq)$ is continuous.

Theorem 6.3. Let $\mathbf{X}$ be a countably-based $T_{0}$-space. The following conditions are equivalent:

- $\mathcal{O}(\mathbf{X})$ is countably-based,

- $\mathcal{O}(\mathbf{X})$ is quasiPolish,

- $\mathbf{X}$ is core-compact.

Proof. As $\mathbf{X}$ is countably-based, $\mathcal{O}(\mathbf{X})$ has the uniform fixed-point property by Theorem 6.1. Therefore, if $\mathcal{O}(\mathbf{X})$ is countably-based then it is continuous and quasiPolish by Theorem 5.1 (note that $\mathcal{O}(\mathbf{X})$ is always $T_{0}$, because the sets $\{U \in \mathcal{O}(\mathbf{X}): x \in U\}$ are Scott open and can separate distinct open sets).

If $\mathcal{O}(\mathbf{X})$ is continuous then the sets $\uparrow U$, where $U \in \mathcal{O}(\mathbf{X})$, form a basis of the Scott topology on $\mathcal{O}(\mathbf{X})$. As $\mathbf{X}$ is countably-based, taking $U$ in the countable family of finite unions of basic open sets induces a countably basis of the Scott topology on $\mathcal{O}(\mathbf{X})$.

\subsection{Non-empty closed sets}

Again, Theorem 4.2 can be proved without the Axiom of Determinacy for spaces of non-empty closed subsets of Polish spaces.

If $\mathbf{X}$ is a represented space, then the set $\mathcal{A}(\mathbf{X})$ of closed subsets of $\mathbf{X}$ has a natural representation: a name of a closed set is simply a name of its complement in $\mathcal{O}(\mathbf{X})$. Therefore, $\mathcal{A}(\mathbf{X}) \cong \mathcal{O}(\mathbf{X})$. Let $\mathcal{A}^{*}(\mathbf{X})$ be the subspace of non-empty closed subsets of $\mathbf{X}$.

Theorem 6.4. Let $\mathbf{X}$ be a Polish space. The following conditions are equivalent:

- $\mathbf{X}$ is compact,

- $\mathcal{A}^{*}(\mathbf{X})$ has the uniform fixed-point property,

- $\mathcal{A}^{*}(\mathbf{X})$ has the fixed-point property.

Proof. Now let $\mathbf{X}$ be a non-compact Polish space. To show that $\mathcal{A}^{*}(\mathbf{X})$ does not satisfy the fixed-point property, it is sufficient by Theorem to prove that in $\mathcal{A}(\mathbf{X}), \mathcal{A}^{*}(\mathbf{X})$ is hard compared to equality, i.e. that equality belongs to $[\Gamma]$ and $\mathcal{A}^{*}(\mathbf{X})$ is $\check{\Gamma}$-hard, for some complexity level $\Gamma$. There are two cases, whether $\mathbf{X}$ is $\sigma$-compact or not.

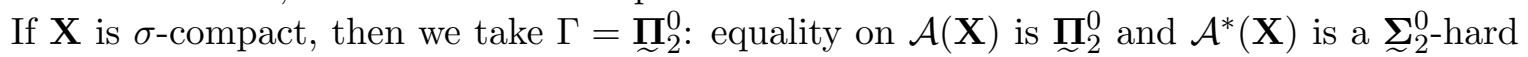
(even complete) subset of $\mathcal{A}(\mathbf{X})$. To show the ${\underset{\sim}{2}}_{2}^{0}$-hardness, one can embed $\mathbb{N}$ as a closed subset of $\mathbf{X}$. It induces an embedding from $\mathcal{A}(\mathbb{N})$ into $\mathcal{A}(\mathbf{X})$, sending $\mathcal{A}^{*}(\mathbb{N})$ to $\mathcal{A}^{*}(\mathbf{X})$. In $\mathcal{A}(\mathbb{N}), \mathcal{A}^{*}(\mathbb{N})$

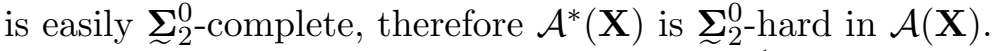

If $\mathbf{X}$ is not $\sigma$-compact then we take $\Gamma=\underset{\sim}{\mathbf{\prod}}$ : equality on $\mathcal{A}(\mathbf{X})$ is $\underset{\sim}{\prod_{1}^{1}}$, and $\mathcal{A}^{*}(\mathbf{X})$ is $\underset{\sim}{\boldsymbol{\Sigma}}{ }_{1}^{1}$-hard (and complete) by applying the same argument as before with $\mathcal{N}$ instead of $\mathbb{N}: \mathcal{N}$ embeds as a 
closed subset of $\mathbf{X}$ (Hurewicz Theorem 7.10 in $[\operatorname{Kec} 95]$ ), so $\mathcal{A}^{*}(\mathcal{N})$ is reducible to $\mathcal{A}^{*}(\mathbf{X})$ and $\mathcal{A}^{*}(\mathcal{N})$ is $\underset{\sim}{\Sigma_{1}^{1} \text {-complete. }}$

Conversely, if $\mathbf{X}$ is compact then $\mathcal{A}^{*}(\mathbf{X})$ is a closed subset, hence a multivalued retract of $\mathcal{A}(\mathbf{X})$. By Proposition 4.2 it is then sufficient to show that $\mathcal{A}(\mathbf{X})$ has the uniform fixed-point property. It is so, because $\mathbf{X}$ is countably-based so $\mathcal{O}(\mathbf{X})$ has the uniform fixed-point property (Theorem 6.1) and $\mathcal{A}(\mathbf{X}) \cong \mathcal{O}(\mathbf{X})$.

\subsection{Functions from Baire space}

In the same way as the recursion theorem is related to the existence of a universal computable function, the fixed-point property for $\mathbf{X}$ is related to the existence of a universal function from $\mathcal{N}$ to $\mathbf{X}$.

Theorem 6.5. Let $\mathbf{X}$ be a represented space. The implications $1 . \Rightarrow 2 . \Rightarrow 3 . \Rightarrow 4$. hold, and are equivalences under the Axiom of Determinacy:

1. $\mathbf{X}$ has the uniform fixed-point property,

2. $\mathcal{C}(\mathcal{N}, \mathbf{X})$ has a total admissible representation,

3. $\mathcal{C}(\mathcal{N}, \mathbf{X})$ is a continuous image of $\mathcal{N}$,

4. $\mathbf{X}$ has the fixed-point property.

Proof. 1. $\Rightarrow 2$. We know from Proposition 4.3 that if $\mathbf{X}$ has the uniform fixed-point property, then so has $\mathcal{C}(\mathcal{N}, \mathbf{X})$, which implies that $\mathcal{C}(\mathcal{N}, \mathbf{X})$ has a total admissible representation.

2. $\Rightarrow 3$. Obvious.

3. $\Rightarrow 4$. It holds by the diagonal argument: if $\phi: \mathcal{N} \rightarrow \mathcal{C}(\mathcal{N}, \mathbf{X})$ is continuous and $h: \mathbf{X} \rightrightarrows \mathbf{X}$ is continuous and fixed-point free, then $f_{0}: \mathcal{N} \rightrightarrows \mathbf{X}$ defined by $f_{0}(p)=h(\phi(p)(p))$ has a continuous selection $f: \mathcal{N} \rightarrow \mathbf{X}$ by Proposition 2.1, which is not in the range of $\phi$. Indeed, for each $p \in \mathcal{N}, f$ differs from $\phi(p)$ on input $p$ : $f(p) \in h(\phi(p)(p))$ so $f(p) \neq \phi(p)(p)$. Therefore, $\phi$ is not a surjection.

4. $\Rightarrow$ 1. It holds under the Axiom of Determinacy, by Theorem 4.2.

Observe that for a general represented space $\mathbf{Y}$, being a continuous image of $\mathcal{N}$ is strictly weaker than having a total admissible representation. For instance, if $\mathbf{Y} \subseteq \mathcal{N}$ or even $\mathbf{Y} \subseteq \mathcal{P}(\omega)$ (i.e. $\mathbf{Y}$ is countably-based) then:

- $\mathbf{Y}$ is a continuous image of $\mathcal{N}$ if and only if $\mathbf{Y} \in \boldsymbol{\Sigma}_{1}^{1}$,

- $\mathbf{Y}$ has a total admissible representation if and only if $\mathbf{Y} \in \underset{\sim}{\mathbf{\Pi}} \mathbf{2}$, i.e. $\mathbf{Y}$ is quasiPolish [dB13].

\section{$7 \quad$ Base complexity}

The latter result Theorem 6.5 has interesting consequences about the base-complexity of topological spaces, introduced and studied in [dBSS16].

Definition 7.1. An admissibly represented space $\mathbf{X}$ is $\mathbf{Y}$-based if there exists a continuous function $\mathbf{Y} \rightarrow \mathcal{O}(\mathbf{X})$ whose range is a basis of the topology on $\mathbf{X}$.

A space $\mathbf{X}$ is $\underset{\sim}{\boldsymbol{\Sigma}} \boldsymbol{\sim}^{1}$-based if it is $\mathbb{N}\langle\alpha\rangle$-based, equivalently if it is $\mathbf{Y}$-based for some $\mathbf{Y} \in \underset{\sim}{\boldsymbol{\Sigma}} \boldsymbol{\alpha}(\mathcal{N})$.

A space $\mathbf{X}$ is countably-based if and only if it is $\mathbb{N}$-based. 
Remark 7.1. A space $\mathbf{X}$ is $\mathcal{N}$-based if and only if $\mathcal{O}(\mathbf{X})$ is a continuous image of $\mathcal{N}$. Indeed, if $\phi: \mathcal{N} \rightarrow \mathcal{O}(\mathbf{X})$ is continuous and its range is a basis of the topology on $\mathbf{X}$, then $\psi: \mathcal{N}^{\mathbb{N}} \rightarrow$ $\mathcal{O}(\mathbf{X})$ sending $p \in \mathcal{N}^{\mathbb{N}}$ to $\bigcup_{i} \phi(p(i))$ is a continuous surjection because $\mathbf{X}$ is hereditarily Lindelöf (the union of every family of open sets is the union of a countable subfamily). Finally observe that $\mathcal{N}^{\mathbb{N}} \cong \mathcal{N}$.

More generally for a countable ordinal $\alpha \geq 1$, a space $\mathbf{X}$ is $\mathbb{N}\langle\alpha\rangle$-based if and only if $\mathcal{O}(\mathbf{X})$ is a continuous image of $\mathbb{N}\langle\alpha\rangle$.

The first result is that there is a gap in the base-complexity of spaces of the form $\mathcal{N} \times \mathbf{X}$ : when they are not countably-based, they are not even $\mathcal{N}$-based.

Corollary 7.1. For an admissibly represented space $\mathbf{X}$, the following conditions are equivalent:

- $\mathbf{X}$ is countably-based,

- $\mathcal{N} \times \mathbf{X}$ is countably-based,

- $\mathcal{N} \times \mathbf{X}$ is $\mathcal{N}$-based.

Proof. The first two conditions are of course equivalent and imply the third one. We know from Theorem 6.1 that $\mathbf{X}$ is countably-based if and only if $\mathcal{O}(\mathbf{X})$ has the (uniform) fixed-point property, which by Theorem 6.5 is equivalent to the property that $\mathcal{C}(\mathcal{N}, \mathcal{O}(\mathbf{X}))$ is a continuous image of $\mathcal{N}$. The latter property precisely means that $\mathcal{N} \times \mathbf{X}$ is $\mathcal{N}$-based, because $\mathcal{C}(\mathcal{N}, \mathcal{O}(\mathbf{X})) \cong$ $\mathcal{O}(\mathcal{N} \times \mathbf{X})$.

Example 7.1. Let us consider the admissibly represented space $\mathbf{X}=\mathcal{N} \times \mathcal{O}(\mathcal{N})$. As both $\mathcal{N}$ and $\mathcal{O}(\mathcal{N})$ are $\mathcal{N}$-based, the product topology on $\mathbf{X}$ is $\mathcal{N}$-based. However, the final topology of the representation on $\mathbf{X}$ is strictly stronger than the product topology, and Corollary 7.1 implies that it is not $\mathcal{N}$-based, as $\mathcal{O}(\mathcal{N})$ is not countably-based.

The previous result implies a first lower bound on the base-complexity of Kleene-Kreisel spaces, which is not optimal.

Corollary 7.2. For any countable ordinal $\alpha \geq 2$, the space $\mathbb{N}\langle\alpha\rangle$ is not $\mathcal{N}$-based.

Proof. $\mathbb{N}\langle\alpha\rangle$ is not countably-based, so $\mathcal{N} \times \mathbb{N}\langle\alpha\rangle$ is not $\mathcal{N}$-based by Corollary 7.1. As $\mathcal{N}$ is a retract of $\mathbb{N}\langle\alpha\rangle, \mathcal{N} \times \mathbb{N}\langle\alpha\rangle$ is a retract of $\mathbb{N}\langle\alpha\rangle \times \mathbb{N}\langle\alpha\rangle \cong \mathbb{N}\langle\alpha\rangle$. As a result, $\mathbb{N}\langle\alpha\rangle$ is not $\mathcal{N}$-based either.

We now identify the base-complexity of the Kleene-Kreisel spaces.

Theorem 7.1 (Base-complexity of Kleene-Kreisel spaces). For any countable ordinal $\alpha \geq 2$, the space $\mathbb{N}\langle\alpha\rangle$ is not $\mathbb{N}\langle\alpha\rangle$-based.

The strategy is to extend Theorem 6.5 and Corollary 7.1, replacing $\mathcal{N}$ with $\mathbb{N}\langle\alpha\rangle$, and apply the diagonal argument using a fixed-point free continuous multifunction $h: \mathcal{O}(\mathbb{N}\langle\alpha\rangle) \rightrightarrows \mathcal{O}(\mathbb{N}\langle\alpha\rangle)$ to prove that $\mathcal{C}(\mathbb{N}\langle\alpha\rangle, \mathcal{O}(\mathbb{N}\langle\alpha\rangle))$ is not a continuous image of $\mathbb{N}\langle\alpha\rangle$. However, it does not work so simply, because previously the diagonal argument relied in an essential way on the possibility of extracting a continuous selection from any continuous multifunction starting from $\mathcal{N}$, because elements of $\mathcal{N}$ have a canonical name (Proposition 2.1). This property is specific to subspaces of $\mathcal{N}$, and not satisfied by $\mathbb{N}\langle\alpha\rangle$. Applying the diagonal argument directly would induce a continuous multifunction from $\mathbb{N}\langle\alpha\rangle$ to $\mathcal{O}(\mathbb{N}\langle\alpha\rangle)$, rather than an element of $\mathcal{C}(\mathbb{N}\langle\alpha\rangle, \mathcal{O}(\mathbb{N}\langle\alpha\rangle))$.

We now show how to overcome this problem.

Definition 7.2. A represented space $\mathbf{X}$ is canonizable if $\mathbf{X}$ contains a set $P$ such that: 
- $P$ is a closed subset of $\mathbf{X}$,

- $\mathbf{X}$ is a continuous image of $P$,

- $P$ is homeomorphic to some subspace of $\mathcal{N}$.

The first property implies that $P$, as a represented subspace of $\mathbf{X}$, has the subspace topology. Thanks to the second property, being a continuous image of $\mathbf{X}$ implies being a continuous image of $P$. The third property exactly says that elements of $P$ have a canonical name, so every continuous multifunction starting from $P$ has a continuous selection (Proposition 2.1).

Example 7.2. $\mathbb{R}$ is canonizable, because it is a continuous image of $\mathbb{N} \times 2^{\mathbb{N}}$ which embeds as a closed subset of $\mathbb{R}$.

The main purpose of this notion is to extend Corollary 7.1 as follows.

Proposition 7.1. Let $\mathbf{X}$ be a canonizable space. If $\mathbf{Y}$ is not countably-based then $\mathcal{O}(\mathbf{X} \times \mathbf{Y})$ is not a continuous image of $\mathbf{X}$.

Proof. Let $P$ make $\mathbf{X}$ canonizable, and let $Q \subseteq \mathcal{N}$ be homeomorphic to $P$. The argument in the proof of Theorem 6.5 can be applied to $Q$ instead of $\mathcal{N}$ and implies that $\mathcal{O}(Q \times \mathbf{Y})$ is not a continuous image of $Q$, hence $\mathcal{O}(P \times \mathbf{Y})$ is not a continuous image of $P$. As $P$ is a closed subset of $\mathbf{X}, \mathcal{O}(P \times \mathbf{Y})$ is a continuous (single-valued) retract, hence a continuous image, of $\mathcal{O}(\mathbf{X} \times \mathbf{Y})$. As $\mathbf{X}$ is a continuous image of $P$, one concludes by transitivity that $\mathcal{O}(\mathbf{X} \times \mathbf{Y})$ is not a continuous image of $\mathbf{X}$.

While this result is an equivalence when $\mathbf{X}=\mathcal{N}$ (Corollary 7.1), it is no more an equivalence in general: Theorem 7.1 implies that for $\mathbf{X}=\mathbb{N}\langle\alpha\rangle$ with $\alpha \geq 2$, the space $\mathcal{O}(\mathbf{X} \times \mathbf{Y})$ is not a continuous image of $\mathbf{X}$, whatever $\mathbf{Y}$ is.

In order to prove Theorem 7.1, we will apply Proposition 7.1 to $\mathbf{X}=\mathbb{N}\langle\alpha\rangle$. We first need to show that it is canonizable.

Lemma 7.1. For every countable ordinal $\alpha, \mathbb{N}\langle\alpha\rangle$ is canonizable.

Proof. The proof is inspired by a construction appearing in the proof of Theorem 7.2 in [SS15a].

We first prove the result for all successor ordinals. $D_{\alpha+1}$ is a $\prod_{\alpha}^{1}$-set, so there exists a continuous surjective function $f: \mathbb{N}\langle\alpha\rangle \rightarrow \mathcal{N} \backslash D_{\alpha+1}$. We define $\psi: D_{\alpha+1} \rightarrow \mathbb{N}\langle\alpha+1\rangle$ as follows. For $x \in D_{\alpha+1}$ and $y \in \mathbb{N}\langle\alpha\rangle$, let $\psi(x)(y)=\min \left\{n \in \mathbb{N}: x_{n} \neq f(y)_{n}\right\}$. $\psi$ is continuous, let $\Psi: D_{\alpha+1} \rightarrow \mathcal{N}$ be a continuous realizer of $\psi$. Let $P \subseteq \mathcal{N} \times \mathbb{N}\langle\alpha+1\rangle$ be the graph of $\psi . P$ is homeomorphic to the graph of $\Psi$ which is a subset of $\mathcal{N} \times \mathcal{N} \cong \mathcal{N}$. $P$ is a closed subset of $\mathcal{N} \times \mathbb{N}\langle\alpha+1\rangle$. Indeed, a pair $(x, F)$ belongs to $P$ iff for all $y \in \mathbb{N}\langle\alpha\rangle$, if $n=F(y)$ then $x_{n} \neq f(y)_{n}$ and $x_{m}=f(y)_{m}$ for all $m<n$, which can be checked for $y$ in some countable dense subset of $\mathbb{N}\langle\alpha\rangle$. If this is not true then one can eventually see it. Finally, $\mathbb{N}\langle\alpha+1\rangle$ is a continuous image of $D_{\alpha+1}$ which is the first projection of $P$.

For limit ordinals, we prove the result by induction. Let $\lambda$ be a limit ordinal. For each each $\beta\left\langle\lambda\right.$, let $P_{\beta}$ witness that $\mathbb{N}\langle\beta\rangle$ is canonizable. By definition, $\mathbb{N}\langle\lambda\rangle=\prod_{i \in \omega} \mathbb{N}\langle\beta(\lambda, i)\rangle$ for some numbering $\beta(\lambda, i)$ of the ordinals smaller than $\lambda$, and one can easily check that $\prod_{i \in \omega} P_{\beta(\lambda, i)}$ makes $\mathbb{N}\langle\lambda\rangle$ canonizable.

We now have all the ingredients to prove Theorem 7.1.

Proof of Theorem 7.1. By Lemma 7.1, $\mathbf{X}=\mathbb{N}\langle\alpha\rangle$ is canonizable. We also know that $\mathbf{Y}=\mathbf{X}$ is not countably-based, so by Proposition 7.1, $\mathcal{O}(\mathbf{X} \times \mathbf{Y})$ is not a continuous image of $\mathbf{X}$. Finally observe that $\mathbf{X} \times \mathbf{Y} \cong \mathbb{N}\langle\alpha\rangle$. 
In order to prove lower bounds on the indexing complexity of certain spaces, we have used three key ingredients. The first two ingredients are clear: the existence of fixed-point free multifunctions, and canonizability. The third ingredient is slightly hidden in the proof and is about extending continuous functions: a continuous function from a closed set $P \subseteq \mathbf{X}$ to $\mathcal{O}(\mathbf{Y})$ extends to a continuous function from $\mathbf{X}$ to $\mathcal{O}(\mathbf{Y})$, simply by sending every $x \notin P$ to the largest open set, namely $\mathbf{Y}$. This extension property is very specific to spaces of open sets. We now give an illustration of the technique on other spaces, enjoying a similar but less obvious extension property.

Proposition 7.2. Let $\alpha$ be a countable ordinal. The spaces $\mathcal{C}(\mathbb{N}\langle\alpha\rangle, \mathbb{R})$ and $\mathcal{C}(\mathbb{N}\langle\alpha\rangle,[0,1])$ are not continuous images of $\mathbb{N}\langle\alpha\rangle$.

Proof. The first case is very easy: there exists a fixed-point free continuous single-valued function $h: \mathbb{R} \rightarrow \mathbb{R}$, namely $h(x)=x+1$, so the standard diagonal argument can be applied: given a continuous function $\phi: \mathbb{N}\langle\alpha\rangle \rightarrow \mathcal{C}(\mathbb{N}\langle\alpha\rangle, \mathbb{R})$ one defines $f \in \mathcal{C}(\mathbb{N}\langle\alpha\rangle, \mathbb{R})$ by $f(x)=h(s(x)(x))=s(x)(x)+1$, which is not in the range of $\phi$, showing that $\phi$ is not surjective.

The second case requires more work: because by the intermediate value theorem, there is no fixed-point free continuous function $h:[0,1] \rightarrow[0,1]$. However, there is a fixed-point free continuous multifunction $h:[0,1] \rightrightarrows[0,1]$ because $[0,1]$ has no least element (more concretely, given a name of a real number $x \in[0,1]$, test in parallel $x>0$ and $x<1$, and ouput 0 or 1 depending on the first test that answers). We then use Schröder's generalization of the Tietze-Urysohn extension theorem [Sch09a], which implies that every continuous function from a functionaly closed subset of $\mathbb{N}\langle\alpha\rangle$ to $[0,1]$ has a continuous extension. A subset of $\mathbb{N}\langle\alpha\rangle$ is functionally closed if it is the zero-set of a continuous function from $\mathbb{N}\langle\alpha\rangle$ to $\mathbb{R}$. It turns out that the closed set $P \subseteq \mathbb{N}\langle\alpha\rangle$ used to show that $\mathbb{N}\langle\alpha\rangle$ is canonizable is functionally closed: it is not hard to see that there is even a continuous function $\eta: \mathbb{N}\langle\alpha\rangle \rightarrow 2^{\mathbb{N}}$ such that $P$ is the preimage of the null sequence under $\eta$.

So given $s: \mathbb{N}\langle\alpha\rangle \rightarrow \mathcal{C}(\mathbb{N}\langle\alpha\rangle,[0,1])$, one defines $f_{0}: P \rightrightarrows[0,1]$ by $f_{0}(x)=h(s(\psi(x)(x))$, where $\psi: P \rightarrow \mathbb{N}\langle\alpha\rangle$ is a continuous surjection. $f_{0}$ is continuous and because $P$ embeds in $\mathcal{N}, f_{0}$ has a continuous choice function $f_{1}: P \rightarrow[0,1]$. By Schröder's generalization of the Tietze-Urysohn extension theorem, $f_{1}$ has a continuous extension $f: \mathbb{N}\langle\alpha\rangle \rightarrow[0,1]$. That function $f$ is not in the range of $s$ : given $x \in \mathbb{N}\langle\alpha\rangle$, let $x_{0} \in P$ be such that $\psi\left(x_{0}\right)=x$, one has $f\left(x_{0}\right)=f_{1}\left(x_{0}\right) \in h\left(s(x)\left(x_{0}\right)\right)$ so $f\left(x_{0}\right) \neq s(x)\left(x_{0}\right)$ and we conclude that $f \neq s(x)$. As it is true for each $x \in \mathbb{N}\langle\alpha\rangle, f$ is not in the range of $s$. Therefore there is no surjection from $\mathbb{N}\langle\alpha\rangle$ to $\mathcal{C}(\mathbb{N}\langle\alpha\rangle,[0,1])$.

\section{Computable indexings}

We now study the effective version of the techniques used in the Section 7. In a represented space $\mathbf{X}$, we are interested in the complexity of enumerating its computable elements $\mathbf{X}_{c}$. Note that $\mathbf{X}_{c}$ is a represented subspace of $\mathbf{X}$.

Definition 8.1. Let $\Gamma$ be a class of subsets of $\mathbb{N}$. A $\Gamma$-indexing of $\mathbf{X}_{c}$ is a computable surjective function from a set $A \in \Gamma$ to $\mathbf{X}_{c}$.

Our goal is to identify, for each $\mathbf{X}$, the minimal indexing complexity of its computable elements.

Example 8.1. The space $\mathcal{P}(\omega)$ has trivial indexing complexity, because there is a total computable enumeration of the c.e. subsets of $\mathbb{N}$. 
Example 8.2. The computable elements of the spaces $\mathcal{N}, 2^{\mathbb{N}}$ and $\mathbb{R}$ have a $\Pi_{2}^{0}$-indexing but no $\Sigma_{2}^{0}$-indexing. Indeed, a $\Sigma_{2}^{0}$-indexing could be turned into a $\Pi_{1}^{0}$-indexing, which could in turn be made total, but there is no total computable enumeration of the computable elements.

We will prove lower bounds on indexing complexity using the same argument, based on canonizability and fixed-point free multifunctions. However we do need to prove their effective versions, which do not follow from previous results. We first need to define the effective version of canonizability.

Definition 8.2. A represented space $\mathbf{X}$ is computably canonizable if $\mathbf{X}$ contains a set $P$ such that:

- $P$ is a $\Pi_{1}^{0}$-subset of $\mathbf{X}$,

- $\mathbf{X}$ is a computable image of $P$,

- $P$ is computably homeomorphic to some subspace of $\mathcal{N}$.

We now prove the effective version of Proposition 7.1, with essentially the same argument. Note however that we need a stronger statement for the applications.

Proposition 8.1. Let $\mathbf{X}$ be computably canonizable and $\mathcal{O}(\mathbf{Y})$ admit a computable fixed-point free multifunction. There is no computable surjection from any $\Pi_{1}^{0}$-subset of $\mathbf{X}$ to $\mathcal{O}(\mathbf{X} \times \mathbf{Y})_{c}$.

Proof. Let $A$ be a $\Pi_{1}^{0}$-subset of $\mathbf{X}$. Let $P \subseteq \mathbf{X}$ witness that $\mathbf{X}$ is computably canonizable and $\phi: P \rightarrow \mathbf{X}$ a computable surjection. Let $Q=\phi^{-1}(A)$. As $Q$ is contained in $P$ which computably embeds in $\mathcal{N}$, we can apply the diagonal argument using the computable fixed-point free multifunction on $\mathcal{O}(\mathbf{Y}): \mathcal{O}(Q \times \mathbf{Y}) \cong \mathcal{C}(Q, \mathcal{O}(\mathbf{Y}))_{c}$ is not a computable image of $Q$. As $Q$ is a $\Pi_{1}^{0}$-subset of $\mathbf{X}, \mathcal{O}(Q \times \mathbf{Y})_{c}$ is a computable image (and even retract) of $\mathcal{O}(\mathbf{X} \times \mathbf{Y})_{c}$, which is therefore not a computable image of $Q$ either. As $A$ is a computable image of $Q, \mathcal{O}(\mathbf{X} \times \mathbf{Y})_{c}$ is not a computable image of $A$.

\subsection{Effective open subsets of Kleene-Kreisel spaces}

We to prove the following effective version of Theorem 7.1.

Theorem 8.1. For $k \in \mathbb{N}$ with $k \geq 2$, there is no $\Sigma_{k}^{1}$-indexing of the effective open subsets of $\mathbb{N}\langle k\rangle$.

We know that for $k \geq 2, \mathbb{N}\langle k\rangle$ is not countably-based so $\mathcal{O}(\mathbb{N}\langle k\rangle)$ admits a fixed-point free continuous multifunction. We show that it even admits a computable one.

Lemma 8.1. For $k \in \mathbb{N}, k \geq 2$, the space $\mathcal{O}(\mathbb{N}\langle k\rangle)$ admits a computable fixed-point free multifunction.

Proof. We prove the result for $k=2$. For $k>2, \mathbb{N}\langle 2\rangle$ is a computable (single-valued) retract of $\mathbb{N}\langle k\rangle$ (Lemma 7.4 in [SS15a]), which it implies that $\mathbb{N}\langle k\rangle$ also admits a computable fixed-point free multifunction.

First observe that for each cylinder $[\sigma] \subseteq \mathcal{N}$, the set $\{F \in \mathbb{N}\langle 2\rangle: F$ is not constantly null on $[\sigma]\}$ is dense. Let $k \in \mathbb{N}$ be larger than the length of $\sigma$ and let $F \in \mathbb{N}\langle 2\rangle$. For each $n \in \mathbb{N}$, let $F_{n} \in \mathbb{N}\langle 2\rangle$ be defined by

$$
F_{n}(p)= \begin{cases}F(p) & \text { if } p(k) \leq n \\ 1 & \text { otherwise }\end{cases}
$$


$F_{n}$ is not constantly null on $[\sigma]$ and $\lim _{n \rightarrow \infty} F_{n}=F$, which shows the claim. Moreover, there exists a computable dense sequence $\left(G_{i}^{\sigma}\right)_{i \in \mathbb{N}}$ in $\mathbb{N}\langle 2\rangle$ such that each $G_{i}$ is not constantly null on $[\sigma]$ (start with all possible $F$ in some fixed computable dense sequence, then rearrange all the $F_{n}$ into one computable sequence).

We now give the algorithm. Given a name of $\mathcal{U} \in \mathcal{O}(\mathbb{N}\langle 2\rangle)$, we compute a function $g \in \mathcal{N}$ as explained below, and output $\mathcal{V}=\{F \in \mathbb{N}\langle 2\rangle: F(g)=0\} \in \mathcal{O}(\mathbb{N}\langle 2\rangle)$.

Start defining $g(0)=0, g(1)=0$, etc. At the same time, test whether $\mathcal{U}$ is non-empty. If that test never halts, then $g$ is the null function. If the test eventually succeeds then at that stage, $g$ is defined up to some input $n$. Let $\sigma=0^{n}$. As the sequence $\left(G_{i}^{\sigma}\right)_{i \in \mathbb{N}}$ is dense, there exists $G_{i}^{\sigma} \in \mathcal{U}$ and $i$ can be effectively found. We can then effectively find some $f \in\left[0^{n}\right]$ such that $G_{i}^{\sigma}(f) \neq 0$, and we let $g=f$.

We now check that $\mathcal{V} \neq \mathcal{U}$. If $\mathcal{U}$ is empty then $\mathcal{V} \neq \mathcal{U}$ as $\mathcal{V}$ is non-empty. If $\mathcal{U}$ is non-empty then one finds $G_{i}^{\sigma} \in \mathcal{U}$ and $G_{i}^{\sigma}(g) \neq 0$, so $G_{i}^{\sigma} \notin \mathcal{V}$ hence $\mathcal{V} \neq \mathcal{U}$.

We know that $\mathbb{N}\langle k\rangle$ is canonizable by Lemma 7.1, but we need the computable version of this notion.

Lemma 8.2. For $k \geq 1, \mathbb{N}\langle k\rangle$ is computably canonizable.

Proof. From Theorem 2.2, as $D_{k} \in \Pi_{k-1}^{1}$ there exists a computable predicate $R \subseteq \mathcal{N} \times \mathbb{N}\langle k-1\rangle \times$ $\mathbb{N}$ such that $x \in D_{k} \Longleftrightarrow \forall y \in \mathbb{N}\langle k-1\rangle, \exists n, R(x, y, n)$. We define a computable function $\psi$ : $D_{k} \rightarrow \mathbb{N}\langle k\rangle$ by $\psi(x)(y)=\min \{n: R(x, y, n)\}$ for $x \in D_{k}, y \in \mathbb{N}\langle k-1\rangle$. Let $P$ be the graph of $\psi$. First, $P$ is a $\Pi_{1}^{0}$-subset of $\mathcal{N} \times \mathbb{N}\langle k\rangle$. Indeed, $(x, H)$ belongs to the graph if and only if for all $y \in \mathbb{N}\langle k-1\rangle$, if $n=H(y)$ then $R(x, y, n)$ and $\neg R(x, y, i)$ for all $i<n$, but this can be checked for all $y$ in a computable dense sequence in $\mathbb{N}\langle k-1\rangle$ (which exists by Lemma 2.1).

As $\psi$ is computable, its graph is computably isomorphic to the graph of a computable realizer of $\psi$, which is a subset of $\mathcal{N} \times \mathcal{N} \cong \mathcal{N}$. Finally, $\mathbb{N}\langle k\rangle$ is a computable image of $D_{k}$ which is the first projection of $P$.

It may possible to extend the result to the constructive ordinals, however we do not address this question in this article.

We can now prove Theorem 7.1. We apply Proposition 8.1 to $\mathbf{X}=\mathbf{Y}=\mathbb{N}\langle k\rangle$, and obtain that $\mathcal{C}(\mathbb{N}\langle k\rangle, \mathcal{O}(\mathbb{N}\langle k\rangle))_{c}$ is not a computable image of any $\Pi_{1}^{0}$-subset of $\mathbb{N}\langle k\rangle$. One has $\mathcal{C}(\mathbb{N}\langle k\rangle, \mathcal{O}(\mathbb{N}\langle k\rangle))_{c} \cong \mathcal{O}(\mathbb{N}\langle k\rangle \times \mathbb{N}\langle k\rangle)_{c} \cong \mathcal{O}(\mathbb{N}\langle k\rangle)_{c}$, and every $\Sigma_{k}^{1}$-subset of $\mathbb{N}$ is a computable image of some $\Pi_{1}^{0}$-subset of $\mathbb{N}\langle k\rangle$ by Theorem 2.2, so $\mathcal{O}(\mathbb{N}\langle k\rangle)_{c}$ has no $\Sigma_{k}^{1}$-indexing.

\subsection{Markov semidecidable properties}

Let $\left(\varphi_{i}\right)_{i \in \mathbb{N}}$ be an effective numbering of the partial computable functions from $\mathbb{N}$ to $\mathbb{N}$.

Definition 8.3. A set $\mathcal{W}$ of total computable functions from $\mathbb{N}$ to $\mathbb{N}$ is Markov semidecidable if there exists a c.e. set $W \subseteq \mathbb{N}$ such that for all $i$ such that $\varphi_{i}$ is total, $i \in W \Longleftrightarrow \varphi_{i} \in \mathcal{W}$.

Every effective open subset of $\mathcal{N}$, restricted to the computable functions, is Markov semidecidable. However, Friedberg [Fri58] exhibited some Markov semidecidable property which is not open. It contrasts with the case of partial computable functions which enjoy the Rice-Shapiro theorem.

Friedberg's example is as follows. If $f_{0}$ is the null function, then the set

$$
\begin{gathered}
\left\{f_{0}\right\} \cup\left\{f: \exists n, \exists i \leq n, \varphi_{i}(0)=f(0)=\ldots=\varphi_{i}(n)=f(n)=0,\right. \\
\left.\varphi_{i}(n+1)=f(n+1) \neq 0\right\}
\end{gathered}
$$


is Markov semidecidable but not open at $f_{0}$.

Understanding what Markov semidecidable properties look like in general, or how to describe them is still an open problem, although some understanding has been developed in [HR17]. Using the techniques developed so far, we provide a step in this direction, identifying the difficulty of indexing these properties. By definition, there is a $\Pi_{3}^{0}$-indexing of them: such a property is given by an index of a Turing machine that for each total computable function, must either accept all its indices or reject all of them, and this statement is $\Pi_{3}^{0}$. We show that it is optimal, which is probably not surprising, but somewhat difficult to prove. This question was left open in [Hoy16].

Theorem 8.2. There is no $\Sigma_{3}^{0}$-indexing of the Markov semidecidable properties of total computable functions.

The rest of the section is devoted to the proof of that result.

We will denote by $\mathcal{N}_{M}$ the represented space of total computable functions from $\mathbb{N}$ to $\mathbb{N}$, where $f \in \mathcal{N}_{M}$ is represented by any index of $f$, i.e. any $i$ such that $f=\varphi_{i}$ (this representation is a partial function from $\mathbb{N}$ to $\mathcal{N}_{M}$, but can be seen as a partial function from $\mathcal{N}$ to $\mathcal{N}_{M}$, because $\mathbb{N}$ computably embeds into $\mathcal{N}$ ). Note that the identity from $\mathcal{N}_{M}$ to $\mathcal{N}_{c}$ is computable, but not its inverse (given an index, one can compute the function, but given the function, one cannot compute an index).

The Markov semidecidable properties are precisely the elements of $\mathcal{O}\left(\mathcal{N}_{M}\right)_{c}$.

Remark 8.1. Type-two computability and Markov computability are very different ways handling computability with infinite objects. However, Markov computability can be seen as an instance of type-two computability and the techniques that we have developed for represented spaces can be applied to Markov computability, as we do in this section.

One still needs to be careful, because the topological concept of admissibility can be misleading here. The final topology of the Markov representation of $\mathcal{N}_{M}$ is the discrete topology, and the Markov representation is admissible for the discrete topology. Therefore as a represented space, $\mathcal{N}_{M}$ is equivalent to $\mathbb{N}$ : there is a bijection between them which is continuously realizable in both directions. However this bijection is not computable, and $\mathcal{N}_{M}$ and $\mathbb{N}$ are very different in terms of computability, as we will see in Lemma 8.4.

The proof of Theorem 8.2 relies on the next three results.

Lemma 8.3. $\mathcal{N}_{M}$ is computably canonizable.

Proof. Let Tot $=\left\{i \in \mathbb{N}: \varphi_{i}\right.$ is total $\}$. If $i \in$ Tot, then let $t_{i}: \mathbb{N} \rightarrow \mathbb{N}$ map $n$ to the halting time of $\varphi_{i}(n)$.

If $i \in \mathbb{N}$ and $f \in \mathcal{N}$, then let $\langle i, f\rangle$ be the function $g \in \mathcal{N}$ sending 0 to $i$ and $n+1$ to $f(n)$. Let $P=\left\{\langle i, t\rangle: \varphi_{i}\right.$ is total and $\left.t=t_{i}\right\} . P$ is a $\Pi_{1}^{0}$-subset of $\mathcal{N}_{M}$ (and even of $\mathcal{N}_{c}$ ). Indeed, one has $\langle i, t\rangle \notin P \Longleftrightarrow \exists n, \varphi_{i}(n)$ does not halt in exactly $t(n)$ steps, which can be eventually discovered.

On $P$, the standard representation and the Markov representation are computably equivalent: given $\langle i, t\rangle \in P$ as a type-two oracle, one can compute an index of $\langle i, t\rangle$, because we know $i$ : it is essentially a program that outputs $i$ on 0 and outputs the halting time of $\varphi_{i}(n)$ on $n+1$. As a result, $P \subseteq \mathcal{N}_{M}$ is computably isomorphic to $P \subseteq \mathcal{N}$.

Finally, $\mathcal{N}_{M}$ is a computable image of the first projection of $P$.

As said in Remark 8.1, the space $\mathcal{N}_{M}$ endowed with the final topology of the Markov representation is homeomorphic to $\mathbb{N}$, so $\mathcal{O}\left(\mathcal{N}_{M}\right)$ is homeomorphic to $\mathcal{P}(\omega)$ and satisfies the uniform fixed-point property. However, $\mathcal{O}\left(\mathcal{N}_{M}\right)_{c}$ does not. 
Lemma 8.4. $\mathcal{O}\left(\mathcal{N}_{M}\right)_{c}$ admits a computable fixed-point free multifunction.

Proof. Let $f_{\infty}$ be the null function, and $f_{k}(n)=0$ if $n \neq k, f_{k}(k)=1$. The first observation is that if $\mathcal{W} \in \mathcal{O}\left(\mathcal{N}_{M}\right)_{c}$ and $f_{\infty} \in \mathcal{W}$, then there exist infinitely many $n$ such that $f_{n} \in \mathcal{W}$. Indeed, there is a computable function $F: \mathbb{N} \rightarrow \mathbb{N}$ such that if $n$ is an index of a program halting in $t$ steps, then $F(n)$ is an index of $f_{t}$, and if $n$ is an index of a non-halting program, then $F(n)$ is an index of $f_{\infty}$. If $f_{n} \notin \mathcal{W}$ for almost every $n$, then the complement of the halting problem would be many-one reducible to $\mathcal{W}$, and would therefore be c.e.

One can think of a name of an element $\mathcal{W}$ of $\mathcal{O}\left(\mathcal{N}_{M}\right)_{c}$ as an enumeration of a set $W \subseteq \mathbb{N}$ containing the indices of the total computable functions that belong to $\mathcal{W}$ and possibly indices of partial functions. Given an enumeration of a set of indices $W$, we enumerate another set $W^{\prime}$ coding some $\mathcal{W}^{\prime} \in \mathcal{O}\left(\mathcal{N}_{M}\right)$ such that $\mathcal{W}^{\prime} \neq \mathcal{W}$.

Given an enumeration of $W$, we enumerate $W^{\prime}$ as follows. Start enumerating all the natural numbers. In parallel, test whether $f_{\infty} \in \mathcal{W}$. If this test eventually succeeds, then let $n$ be larger than all the numbers enumerated so far in $W^{\prime}$. Look for $n+1$ distinct values of $k$ such that $f_{k} \in \mathcal{W}$, and let $K$ be larger than all of them. Now enumerate in $W^{\prime}$ all the numbers $j$ such that $\varphi_{j}$ coincides with some $\varphi_{l}, l<n$, on inputs $0,1, \ldots, K$.

Now we check that the construction fulfills the conditions.

- If $f_{\infty} \notin \mathcal{W}$ then $W^{\prime}=\mathbb{N}$ and $\mathcal{W}^{\prime}=\mathcal{N}_{c}$, so $f_{\infty} \in \mathcal{W}^{\prime} \backslash \mathcal{W}$.

- If $f_{\infty} \in \mathcal{W}$ then there exist infinitely many $k$ 's such that $f_{k} \in \mathcal{W}$, so the algorithm will eventually find $K$, and $\mathcal{W}^{\prime}$ is the set of functions that coincide with some $\phi_{l}, l<n$, on inputs $0,1, \ldots, K$. We show that some $f_{k}$ belongs to $\mathcal{W} \backslash \mathcal{W}^{\prime}$. Indeed, by the choice of $K$, there are at least $n+1$ values of $k<K$ such that $f_{k} \in \mathcal{W}$, and all these functions differ on some input $\leq K$. By the pigeonhole principle, one of them must differ from each $\varphi_{l}, l<n$, on some input $\leq K$. As result, $f_{k} \in \mathcal{W} \backslash \mathcal{W}^{\prime}$.

Lemma 8.5. For a set $A \subseteq \mathbb{N}$, the following conditions are equivalent:

1. A is a computable image of some $\Pi_{1}^{0}$-subset of $\mathcal{N}_{c}$,

2. A is a computable image of some $\Pi_{1}^{0}$-subset of $\mathcal{N}_{M}$,

3. $A \in \Sigma_{3}^{0}(\mathbb{N})$.

Proof. 1. $\Rightarrow 2$. It is straightforward, because the identity from $\mathcal{N}_{M}$ to $\mathcal{N}_{c}$ is computable, so every $\Pi_{1}^{0}$-subset of $\mathcal{N}_{c}$ is a $\Pi_{1}^{0}$-subset of $\mathcal{N}_{M}$.

2 . $\Rightarrow 3$. It follows from the fact that $\mathcal{N}_{M}$, hence any $\Pi_{1}^{0}$-subset of it, has a $\Pi_{2}^{0}$-indexing.

3. $\Rightarrow 1$. It is sufficient to prove it for $A \in \Pi_{2}^{0}(\mathbb{N})$, because $\Sigma_{3}^{0}$-sets are computable images of $\Pi_{2}^{0}$-sets. Let $A=\bigcap_{i} \bigcup_{j} A_{i, j}$ where $\left(A_{i, j}\right)_{i, j \in \mathbb{N}}$ is a computable double-sequence of finite subsets of $\mathbb{N}$. Let $P=\left\{(n, f) \in \mathbb{N} \times \mathcal{N}_{c}: \forall i, n \in A_{i, f(i)}\right\}$. $P$ is a $\Pi_{1}^{0}$-subset of $\mathbb{N} \times \mathcal{N}_{c} \cong \mathcal{N}_{c}$ and its first projection is $A$.

Strictly speaking we cannot apply Proposition 8.1. But the same argument shows that there is no computable surjection from any $\Pi_{1}^{0}$-subset of $\mathcal{N}_{M}$ to $\mathcal{C}\left(\mathcal{N}_{M}, \mathcal{O}\left(\mathcal{N}_{M}\right)_{c}\right)_{c} \cong \mathcal{O}\left(\mathcal{N}_{M}\right)_{c}$.

Finally, by Lemma 8.5, every $\Sigma_{3}^{0}$-subset of $\mathbb{N}$ is a computable image of a $\Pi_{1}^{0}$-subset of $\mathcal{N}_{M}$, so there is no $\Sigma_{3}^{0}$-indexing of $\mathcal{O}\left(\mathcal{N}_{M}\right)_{c}$. 


\section{References}

[BG21] Vasco Brattka and Guido Gherardi. Completion of choice. Ann. Pure Appl. Log., 172(3):102914, 2021.

[BGng] Vasco Brattka and Guido Gherardi. Weihrauch goes Brouwerian. Journal of Symbolic Logic, pages 1-36, forthcoming.

[BH94] Vasco Brattka and Peter Hertling. Continuity and computability of relations. Informatik Berichte 164, FernUniversität Hagen, Hagen, September 1994.

[Bra20] Vasco Brattka. The discontinuity problem. Available at arXiv:2012.02143, 2020.

[CH20] Antonin Callard and Mathieu Hoyrup. Descriptive complexity on non-Polish spaces. In Christophe Paul and Markus Bläser, editors, 37th International Symposium on Theoretical Aspects of Computer Science, STACS 2020, March 10-13, 2020, Montpellier, France, volume 154 of LIPIcs, pages 8:1-8:16. Schloss Dagstuhl - LeibnizZentrum für Informatik, 2020.

[dB13] Matthew de Brecht. Quasi-Polish spaces. Ann. Pure Appl. Logic, 164(3):356-381, 2013.

[dBSS16] Matthew de Brecht, Matthias Schröder, and Victor Selivanov. Base-complexity classifications of qcbo-spaces. Computability, 5(1):75-102, 2016.

[Ern91] Marcel Erné. The ABC of order and topology. In Category Theory at Work, Proceedings of a Workshop, volume 18 of Research and Exposition in Mathematics, pages 57-83. Heldermann, 1991.

[Fri58] Richard M. Friedberg. Un contre-exemple relatif aux fonctionnelles récursives. Comptes Rendus de l'Académie des Sciences, 247:852-854, 1958.

[GL13] Jean Goubault-Larrecq. Non-Hausdorff Topology and Domain Theory: Selected Topics in Point-Set Topology. New Mathematical Monographs. Cambridge University Press, 2013.

[Hoy16] Mathieu Hoyrup. The decidable properties of subrecursive functions. In ICALP 2016, July 11-15, 2016, Rome, Italy, volume 55 of LIPIcs, pages 108:1-108:13. Schloss Dagstuhl - Leibniz-Zentrum fuer Informatik, 2016.

[Hoy20] Mathieu Hoyrup. Descriptive complexity on non-polish spaces II. In Artur Czumaj, Anuj Dawar, and Emanuela Merelli, editors, 47 th International Colloquium on Automata, Languages, and Programming, ICALP 2020, July 8-11, 2020, Saarbrücken, Germany (Virtual Conference), volume 168 of LIPIcs, pages 132:1-132:17. Schloss Dagstuhl - Leibniz-Zentrum für Informatik, 2020.

[HR17] Mathieu Hoyrup and Cristobal Rojas. On the information carried by programs about the objects they compute. Theory Comput. Syst., 61(4):1214-1236, 2017.

[Hyl79] J.M.L. Hyland. Filter spaces and continuous functionals. Annals of Mathematical Logic, 16:101-143, 1979.

[Kec95] Alexander S. Kechris. Classical Descriptive Set Theory. Springer, January 1995. 
[KW85] Christoph Kreitz and Klaus Weihrauch. Theory of representations. Theoretical Computer Science, 38:35 - 53, 1985.

[Law69] F. William Lawvere. Diagonal arguments and cartesian closed categories, pages 134145. Springer Berlin Heidelberg, Berlin, Heidelberg, 1969.

[Nor80] D. Normann. Recursion on the Countable Functionals. Lecture Notes in Mathematics. Springer Berlin Heidelberg, 1980.

[NP19] Hugo Nobrega and Arno Pauly. Game characterizations and lower cones in the weihrauch degrees. Log. Methods Comput. Sci., 15(3), 2019.

[Oxt13] J.C. Oxtoby. Measure and Category. Graduate Texts in Mathematics. Springer New York, 2013.

[Pau16] Arno Pauly. On the topological aspects of the theory of represented spaces. Computability, 5(2):159-180, 2016.

[PdB15] Arno Pauly and Matthew de Brecht. Descriptive set theory in the category of represented spaces. In 30th Annual ACM/IEEE Symposium on Logic in Computer Science, LICS 2015, Kyoto, Japan, July 6-10, 2015, pages 438-449. IEEE Computer Society, 2015 .

[PZ13] Arno Pauly and Martin Ziegler. Relative computability and uniform continuity of relations. J. Log. Anal., 5, 2013.

[Sch02] Matthias Schröder. Admissible Representations for Continuous Computations. PhD thesis, FernUniversität Hagen, 2002.

[Sch04] Matthias Schröder. Spaces allowing type-2 complexity theory revisited. Math. Log. Q., 50(4-5):443-459, 2004.

[Sch09a] Matthias Schröder. An effective Tietze-Urysohn theorem for QCB-spaces. Journal of Universal Computer Science, 15(6):1317-1336, mar 2009.

[Sch09b] Matthias Schröder. The sequential topology on $\mathbb{N}^{\mathbb{N}}$ is not regular. Mathematical Structures in Computer Science, 19(5):943-957, 2009.

[Sch12] Bernd S. W. Schröder. The fixed point property for ordered sets. Arabian Journal of Mathematics, 1:529-547, 2012.

[Sch15] Matthis Schröder. A Hofmann-Mislove theorem for Scott open sets. Available at arXiv:1501.06452, 2015.

[SS15a] Matthias Schröder and Victor Selivanov. Some hierarchies of qcb 0 -spaces. Mathematical Structures in Computer Science, 25(8):1799-1823, 2015.

[SS15b] Matthias Schröder and Victor L. Selivanov. Hyperprojective hierarchy of qcb 0 -spaces. Computability, 4(1):1-17, 2015.

[Wei00] Klaus Weihrauch. Computable Analysis. Springer, Berlin, 2000. 